\title{
Early and Late Effects of Maternal Experience on Hippocampal Neurogenesis, Microglia, and the Circulating Cytokine Milieu
}

Rand S Eid ${ }^{1}$, Jessica A Chaiton², Stephanie E Lieblich², Tamara S Bodnar ${ }^{3}$, Joanne Weinberg ${ }^{1,3}$, and Liisa AM Galea ${ }^{1,2,4}$

${ }^{1}$ Graduate Program in Neuroscience, University of British Columbia, Vancouver, BC, Canada

${ }^{2}$ Department of Psychology, University of British Columbia, Vancouver, BC, Canada

${ }^{3}$ Department of Cellular and Physiological Sciences, University of British Columbia, Vancouver, BC, Canada

${ }^{4}$ Djavad Mowafaghian Centre for Brain Health, University of British Columbia, Vancouver, BC, Canada

Corresponding author:

Dr. Liisa Galea

Djavad Mowafaghian Centre for Brain Health

University of British Columbia

2215 Wesbrook Mall

Vancouver, BC

Canada, V6T $1 Z 3$

Tel: +1 (604) 8226536

Email: liisa.galea@ubc.ca 


\section{Abstract}

2 The maternal brain displays considerable plasticity, and motherhood is associated with changes in

3 affective and cognitive function. Motherhood can alter the trajectory of brain ageing, including

4 modifications to neuroplasticity and cognition. Here, we investigated the short- and long-term effects of

5 motherhood on hippocampal neurogenesis, microglial density and morphology, and circulating

6 cytokines, domains known to be altered with age and implicated in cognition and mood. Female rats

7 were bred then euthanized during gestation or at various postpartum timepoints, culminating in middle

8 age, and nulliparous rats served as age-matched controls. Hippocampal neurogenesis was significantly

9 suppressed during gestation and the postpartum period. Interestingly, neurogenesis declined significantly

10 in middle-aged nulliparous rats, but increased in primiparous rats across the same period. Transient

11 postpartum adaptations to the neuroimmune environment of the hippocampus were evidenced, as Iba-1-

12 immunoreactive microglia assumed a de-ramified morphology followed by increased density.

13 Intriguingly, ageing-related changes in circulating cytokines were dependent on parity. These

14 adaptations in neurogenic and immune processes may have ramifications for maternal mood and

15 cognition across the peripartum period and beyond.

16 Keywords: pregnancy; postpartum; motherhood; doublecortin; cell proliferation; Iba-1;

17 proinflammatory cytokines; anti-inflammatory cytokines. 


\section{Introduction}

19 Dramatic physiological adaptations occur during pregnancy and the postpartum period to ensure offspring development and survival (Dulac et al., 2014; Hall et al., 2011; Lain and Catalano, 2007; PrabhuDas et al., 2015; Rossant and Cross, 2001). The maternal brain exhibits substantial plasticity,

22 including large-scale volumetric changes (Galea et al., 2000; Hoekzema et al., 2016; Oatridge et al., 23 2002), alterations in cellular architecture (Leuner and Gould, 2010; Pawluski and Galea, 2006), and

24 hippocampal neurogenesis (Darnaudéry et al., 2007; Leuner et al., 2007; Pawluski and Galea, 2007).

25 While this capacity for plasticity is likely essential for the onset of a repertoire of maternal behaviours 26 (Bridges, 2015), motherhood is also associated with changes in affective function (Bennett et al., 2004;

27 Darcy et al., 2011; O’Hara, 2009), hypothalamic-pituitary-adrenal axis (HPA) regulation (Slattery and Neumann, 2008), and cognition (Cuttler et al., 2011; De Groot et al., 2006; Galea et al., 2000; Kinsley et al., 1999; Pawluski et al., 2006). Interestingly, motherhood may improve the ageing trajectory in terms of cognition (Colucci et al., 2006; Cui et al., 2014; Gatewood et al., 2005), neuroplasticity (Barha et al., 2015; Barha and Galea, 2011; Galea et al., 2018), and cellular aging (Barha et al., 2016), suggesting that the effects of the motherhood on the brain may be long lasting. However, the mechanisms underlying alterations in the ageing maternal brain are not known, but may include modifications in neurogenic or immune processes, both of which were examined in the current study.

The hippocampus produces new neurons across the lifespan (Altman and Das, 1965; Boldrini et al., 2018; Eriksson et al., 1998) and these neurons play a role in certain aspects of learning and memory (Yau et al., 2015), mood regulation (Sahay and Hen, 2007), and the stress response (Snyder et al., 2011). Importantly, several studies found postpartum reductions in hippocampal cell proliferation (Darnaudéry et al., 2007; Leuner et al., 2007; Pawluski and Galea, 2007), cell survival (Pawluski and Galea, 2007) and the density of immature neurons (Workman et al., 2015). Interestingly, motherhood may have contrasting effects on hippocampal neurogenesis with age, as studies have found increased neurogenesis in middle-aged primiparous and multiparous rats relative to age-matched nulliparous controls (Barha et al., 2015; Galea et al., 2018). This finding signifies that parity can have delayed pro-neurogenic effects, thus the current study aimed to determine the timeline by which these changes may emerge.

Adaptations to the maternal immune system are well documented, and necessary for the establishment and maintenance of pregnancy (Aghaeepour et al., 2017; Mor and Cardenas, 2010). In

47 contrast, and despite the growing recognition of plasticity in the maternal brain, there is a paucity of 48 research on potential neuroimmune adaptations with maternal experience. Few studies to date have 
examined microglia, the innate immune cells of the brain, in pregnant and postpartum rats (Haim et al., 2017; Posillico and Schwarz, 2016). Microglia alterations were found in several regions of the maternal brain, and normalized by postpartum day 21 in all regions except the hippocampus (Haim et al., 2017). This indicates that changes in the neuroimmune environment of the maternal hippocampus may be

53 longer lasting. The hippocampus undergoes considerable plasticity in the peripartum period (Galea et al., 2014), perhaps not surprisingly given its role in cognitive function (Sweatt, 2004) and mood regulation (Campbell and MacQueen, 2004). Neuroimmune processes are implicated in cognition (Lee et al., 2008; Parkhurst et al., 2013; vom Berg et al., 2012), stress (Hodes et al., 2014; Kreisel et al., 2014), and mood (Setiawan et al., 2015), raising the possibility that changes in the neuroimmune environment of the hippocampus may represent a substrate for motherhood-related changes in hippocampal function.

In the non-maternal brain, immune processes have been implicated in the regulation of adult hippocampal neurogenesis, under basal and inflammatory conditions (reviewed in Sierra et al., 2014). For example, inflammation was first demonstrated to suppress neurogenesis by studies utilizing systemic or intrahippocampal administration of the bacterial endotoxin lipopolysaccharide (Ekdahl et al., 2003; Monje, 2003). Microglia also maintain homeostasis in the healthy adult neurogenic niche via phagocytosis of apoptotic new cells (Sierra et al., 2010). In the maternal brain, one study found that alterations in T-cell activity accounted for at least some of the postpartum-associated reductions in neurogenesis (Rolls et al., 2008). To date, however, no studies have concurrently examined adaptations in microglia and neurogenesis in the maternal hippocampus, and therefore the experiments reported here aimed to fill this gap.

Given the extensive cross-talk between the central nervous system and the immune system (Louveau et al., 2015), motherhood-related adaptations in the immune system can potentially drive plasticity in the brain. Reproductive immunology research has been primarily focused on aspects of

72 immune function that affect fetal development and the success of pregnancy (PrabhuDas et al., 2015).

73 Although many of the maternal immune adaptations normalize in the postpartum period (Groer et al.,

74 2015), there is evidence indicating that maternal experience can leave a lasting footprint on the immune 75 system (Barrat et al., 1997a, 1997b, 1999; Helle et al., 2004). For example, the risk of dying of 76 infectious disease after the age of 65 was increased in mothers of twins, compared to mothers of 77 singletons (Helle et al., 2004). This effect may be related to reproductive effort, and is perhaps indicative 78 of accelerated immunosenescence (Helle et al., 2004). Although the long-term effects of parity on the 79 immune system have received little attention in animal models, delayed senescence in certain aspects of 80 immune function is evidenced in parous relative to non-parous mice (Barrat et al., 1997b, 1997a, 1999). 
81 In tandem with neurogenic and neuroimmune markers, our current study aimed to assess whether

82 maternal experience can alter the circulating cytokine profile at various intervals following parturition,

83 ending well after the reproductive event itself. The circulating cytokine profile is not only informative to

84 the general inflammatory state, but may have ramifications for brain and behaviour as peripheral

85 cytokine signals propagate to the brain (Miller et al., 2014; Quan and Banks, 2007). Preclinical cytokine

86 data may also be valuable for comparative purposes, as circulating cytokines levels are accessible

87 biomarkers in clinical populations (Guerreiro et al., 2007).

In this study, we examined the short- and long-term effects of parity on microglia density and morphology, and on neurogenesis in the hippocampus. These measures were examined across age and

91 milieu, we quantified concentrations of various serum cytokines across the same time points. We expected parity to suppress hippocampal neurogenesis in the short term, and to increase neurogenesis in

93 middle age. At least in the short term, we expected parity to modify microglial density and morphology

94 in the dentate gyrus. Finally, we expected alterations in the circulating cytokine profile during pregnancy 95 and the early postpartum period, and hypothesized that parity would modulate the age-related changes in 96 the circulating cytokine milieu.

\section{2. Materials and Methods}

\subsection{Animals}

99 Young adult female and male Sprague-Dawley rats were purchased from Charles River Laboratories 100 (Montreal, Canada), weighing at 200-250g. All rats arrived at our facility at the same time. Rats were 101 maintained on a 12-hour light/dark cycle (lights on at 07:00 h), in standard laboratory conditions $102\left(21 \pm 1{ }^{\circ} \mathrm{C} ; 50 \pm 10 \%\right.$ humidity) and given ad libitum access to water and food (Purina Rat Chow).

103 Female rats were initially pair-housed, and except for the breeding period, all rats were housed in 104 female-only colony rooms. Males were used for breeding purposes only. Nulliparous rats were never 105 housed in the same colony room as primiparous rats when they were breeding or had active litters. To 106 minimize potential environmental exposure differences between nulliparous and primiparous groups, 107 primiparous rats were transferred to the nulliparous colony room on the day that their litters were 108 weaned (postpartum day 21). All procedures were performed in accordance with ethical guidelines set 109 by the Canadian Council on Animal Care and approved by the Animal Care Committee at the University 110 of British Columbia. 


\section{2.2. Breeding procedure and experimental groups}

112 Female rats were bred at approximately 7 months of age. At 18:00 h daily, each pair of female cage113 mates was placed with one male. Vaginal lavage samples were obtained the following morning, between 114 08:00 and 09:00 h, and examined for the presence of sperm cells. The detection of sperm cells indicated 115 Gestation Day 1 (GD1), at which point the pregnant female was weighed and single-housed. Primigravid 116 or primiparous rats (i.e. pregnant or mothering for the first time; $n=30$ ) were randomly assigned to one 117 of six groups ( $\mathrm{n}=5$ each) according to the timeline of euthanasia relative to gestation. This included one 118 gestational group at Gestation Day 13 (GD13), and four postpartum groups: Postpartum Day 8 (PPD8), 119 Postpartum Day 30 (PPD30), Postpartum Day 90 (PPD90), and Postpartum Day 180 (PPD180).

120 Nulliparous rats (i.e. never pregnant; $n=30$ ) were randomly assigned to control groups ( $n=5$ each) that 121 were age-matched to each of the primiparous groups. Specifically, nulliparous rats at approximately 7, 122 7.5, 8, 10, and 13 months of age served as control groups for primiparous rats at GD13, PPD8, PPD30, 123 PPD90, and PPD180, respectively (experimental groups are detailed in Figure 1). These timepoints 124 were chosen to capture: mid-gestation (GD13), as a previous study found reductions in the survival of 125 hippocampal neurons produced at this time (Rolls et al., 2008); an early postpartum timepoint (PPD8) 126 that avoids the acute inflammatory state surrounding parturition (Catalano et al., 2010) and is associated 127 with declines in cell proliferation (Leuner et al., 2007); a post-weaning, late postpartum timepoint 128 (PPD30) shown to be associated with reduced neurogenesis (Workman et al., 2015); and finally, for a 129 time-course analysis of the effects of parity on the ageing trajectory, two further timepoints were 130 selected leading to middle age (PPD180), as previous studies reported increased neurogenesis in middle131 aged rats with previous maternal experience (Barha et al., 2015; Galea et al., 2018). To account for 132 potential effects of social housing, nulliparous controls were single-housed at GD1 of their primiparous133 counterparts and until experimental endpoint. For all postpartum groups, litters were culled to include 134 between 8-10 pups, with approximately 50\% males and females. When the original sex ratio or litter size 135 was not sufficient to achieve this, pups were cross-fostered between dams that had given birth the same 136 day. Pups were weaned at PPD21 for all postpartum groups, except for the PPD8 group in which the 137 dams remained with their litter until just prior to euthanasia. After weaning, primiparous rats remained 138 single-housed until experimental endpoint. One rat from the nulliparous 10-month-old group was 139 eliminated from the study due to a mammary gland tumor. 


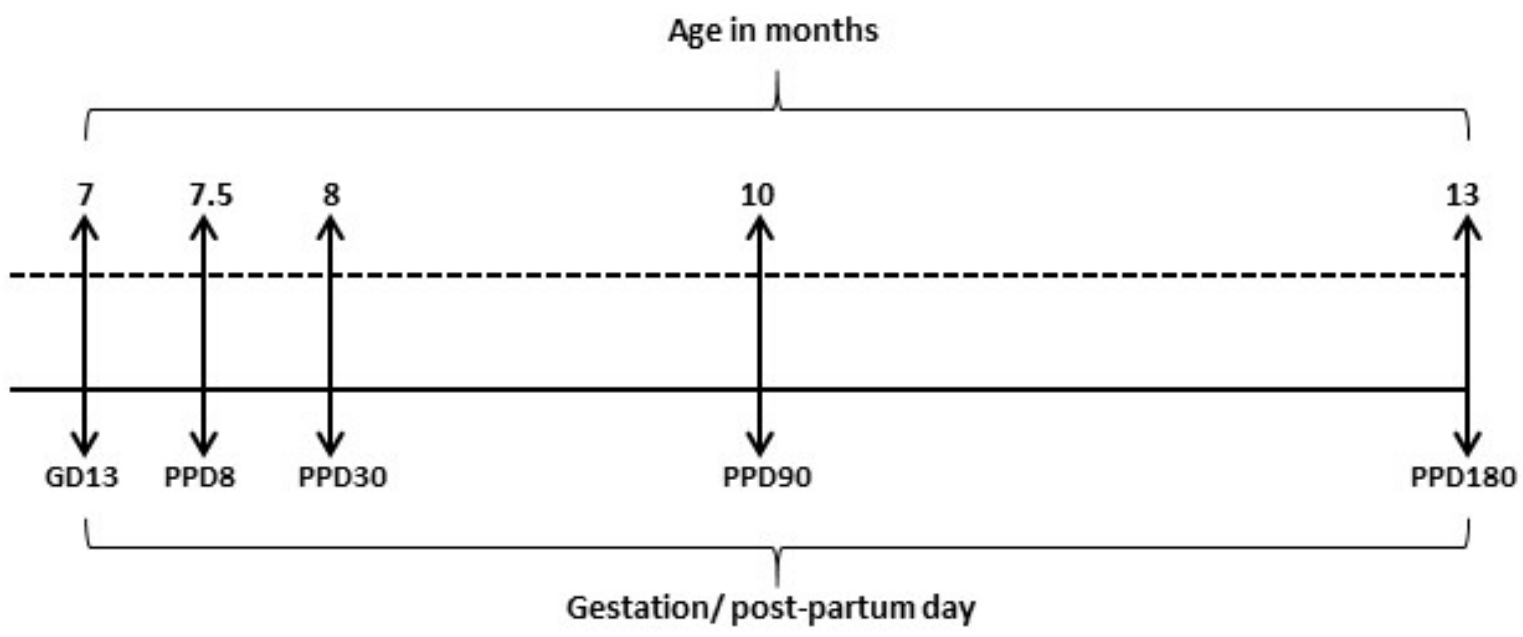

Figure 1. Experimental groups. Primigravid or primiparous rats ( $\mathrm{n}=5$ /group) were euthanized at gestation day 13 (GD13), postpartum day 8 (PPD8), postpartum day 30 (PPD30), postpartum day 90 (PPD90), or postpartum day 180 (PPD180). Nulliparous rats (n=5/group) were age-matched to their primiparous counterparts, and euthanized along the same timeline, depicted as approximate age in months. Arrows indicate euthanasia day.

\subsection{Perfusion and tissue collection}

141 All perfusions were completed between 9:00 and 11:00 am. The rats were deeply anesthetized with an 142 overdose of sodium pentobarbital (i.p.), and blood was collected via cardiac puncture. Brains were 143 collected immediately after transcardial perfusion with $60 \mathrm{ml}$ of cold $0.9 \%$ saline, followed by $120 \mathrm{ml}$ of 144 cold $4 \%$ paraformaldehyde (PFA). Brains were stored at $4^{\circ} \mathrm{C}$ in $4 \%$ PFA for 24 hours, then transferred 145 into a 30\% sucrose solution (in 0.1 M Phosphate Buffer) until sectioning. In the group euthanized during 146 gestation, the uterus was dissected to confirm pregnancy. To obtain serum, blood samples were allowed 147 to clot overnight at $4^{\circ} \mathrm{C}$, then centrifuged at $10 \mathrm{~g}$ for 15 minutes and serum aliquots were stored at $-20^{\circ} \mathrm{C}$ 148 until processing.

\section{2.4. Brain Tissue Processing and Immunohistochemistry}

150 Brains were sliced into $40 \mu \mathrm{m}$ coronal sections using a Leica SM2000R Microtome (Richmond Hill, 151 Ontario, Canada). Sections were collected in series of 10 along the rostral-caudal axis of the 152 hippocampus, then stored at $-20^{\circ} \mathrm{C}$ in a cryoprotectant consisting of $30 \%$ ethylene glycol (Sigma153 Aldrich, St. Louis, MO, USA) and 20\% glycerol (Sigma-Aldrich) in 0.1 M phosphate-buffer (PB, pH 154 7.4). Sections were thoroughly rinsed (5 x 10 mins) in PBS prior to staining to remove the 155 cryoprotective medium. All immunohistochemical procedures were conducted on free-floating brain 156 sections, and on a rotator at room temperature unless otherwise noted. 
157 2.4.1. Doublecortin (DCX): DCX is a microtubule-associated protein expressed in immature neurons for

15821 days after production in adult rats (Brown et al., 2003), and thus was used as a marker of adult

159 hippocampal neurogenesis. Tissue was rinsed in 0.1M PBS (pH 7.4; 5 x 10 minutes) between each of the

160 following procedures. Tissue was treated with $0.3 \%$ hydrogen peroxide $\left(\mathrm{H}_{2} \mathrm{O}_{2}\right.$, in $\left.\mathrm{dH}_{2} \mathrm{O}\right)$ for 30 minutes,

161 then incubated for 24 hours at $4^{\circ} \mathrm{C}$ in a primary antibody solution containing 1:1000 goat anti-

162 doublecortin (Santa Cruz Biotechnology, Santa Cruz, CA, USA) in 3\% normal rabbit serum (NRS) and

$1630.4 \%$ Triton-X in 0.1M PBS. Next, tissue was transferred to a secondary antibody solution consisting of

164 1:500 rabbit anti-goat (Vector Laboratories, Burlington, ON, Canada) in $0.1 \mathrm{M}$ PBS for 24 hours at $4^{\circ} \mathrm{C}$.

165 Finally, tissue was transferred to an avidin-biotin complex (ABC; Elite kit; 1:1000, Vector Laboratories)

166 in PBS for 4 hours, then immunoreactants were visualized with a Nickel-enhanced DAB reaction

167 (Vector Laboratories). Sections were mounted onto glass slides and allowed to dry, then dehydrated in

168 increasing graded ethanol, defatted with xylenes, and cover-slipped with Permount (Fisher Scientific).

169 2.4.2. Ionized calcium binding adaptor molecule-1 (Iba-1): Iba-1 is a calcium-binding protein widely

170 used as a microglial marker (Korzhevskii and Kirik, 2016). Tissue was rinsed in 0.1 M PBS (pH 7.4; 3 x

17110 minutes) between each of the following procedures. Tissue was incubated in 0.3\% hydrogen peroxide

$172\left(\mathrm{H}_{2} \mathrm{O}_{2}\right.$, in $\left.\mathrm{dH}_{2} \mathrm{O}\right)$ for 25 minutes, then blocked with $10 \%$ normal goat serum (NGS) in $0.5 \%$ Triton-X in

$1730.1 \mathrm{M}$ PBS. Tissue was then transferred to a primary antibody solution for 18 hours at $4^{\circ} \mathrm{C}$, consisting of

174 1:1000 anti-Iba-1 (Wako, Osaka, Japan) in 10\% NGS and 0.4\% Triton-X in 0.1M PBS. Next, tissue was

175 incubated in a secondary antibody solution for 1 hour, containing 1:500 biotinylated anti-rabbit (Vector

176 Laboratories) in 2.5\% NGS and 0.4\% Triton X in PBS. Finally, tissue was transferred to an avidin-biotin

177 complex (ABC; Elite kit; 1:50, Vector Laboratories) in 0.4\% Triton-X in PBS for 1 hour, and

178 immunoreactivity was visualized with a Nickel-enhanced DAB reaction (Vector Laboratories). Sections

179 were mounted onto glass slides and allowed to dry, then counterstained with cresyl violet, dehydrated in

180 a series of ethanol solutions of increasing concentrations, defatted with xylenes, and cover-slipped with

181 Permount (Fisher Scientific).

182 2.4.3. Ki67: Ki67 is expressed during all active phases of the cell cycle, but not during $\mathrm{G}_{0}$ phase

183 (Scholzen and Gerdes, 2000), and therefore was used as a marker of cell proliferation in the dentate

184 gyrus. Tissue was rinsed in 0.1 M PBS (pH 7.4; 3 x 10 minutes) between each of the following

185 procedures. Tissue was incubated in a primary antibody solution for 48 hours at $4^{\circ} \mathrm{C}$, consisting of $1: 200$

186 mouse anti-Ki67 (NCL-L-Ki67-MM1; Leica Biosystems, Newcastle, UK) in 3\% normal donkey serum

187 (NDS), and $0.3 \%$ Triton-X in $0.1 \mathrm{M}$ PBS. Next, tissue was incubated for 18 hours at $4^{\circ} \mathrm{C}$ in a secondary

188 antibody solution consisting of 1:200 donkey anti-mouse IgG, Alexa Fluor 555 (Molecular Probes, 
189 Eugene, Oregon, USA) in 3\% NDS in 0.1M PBS. Sections were counterstained with DAPI (2.5 minutes;

190 300nM; ThermoFisher, Waltham, WA, USA), mounted onto glass slides, and cover-slipped with an anti-

191 fade medium (2.5\% Polyvinyl alcohol-Dabco).

\subsection{Microscopy, Cell Quantification, and Morphological analyses}

193 An investigator blinded to experimental conditions quantified DCX- and Iba-1-, and Ki67-

194 immunoreactive cells and analyzed cell morphology. See Fig. 4B, 6D-F, and 7 for representative 195 photomicrographs.

2.5.1. Iba-1: Under a 40x objective on a Nikon E600 microscope, an exhaustive quantification of Iba-1-

197 IR cells was completed in four hippocampal slices from each animal, as we have done previously

198 (Mahmoud et al., 2016a). This included two dorsal and two ventral sections, with approximate Bregma

199 coordinates of $-3.12,-3.48,6.00$, and -6.36. Iba-1-IR cells were quantified in the dentate gyrus,

200 specifically in the granule cell layer (GCL), the subgranular zone (SGZ), and within an approximately

$20150 \mu \mathrm{m}$ band of the molecular layer (ML).

202 Under baseline conditions microglia typically display ramified morphology, characterized by highly

203 branched and long processes that continuously survey their environment (Nimmerjahn et al., 2005).

204 Under inflammatory states, microglial processes typically retract and the cell can take on an amoeboid

205 morphology, in which the cell become enlarged and void of processes; thus, microglial morphology is

206 widely used as a proxy-measure of functional state (Karperien et al., 2013). Here, Iba-1-IR cell

207 morphology was analyzed utilizing NIS Elements Basic Research software (Nikon) under a Nikon E600

208 microscope. Using the measure feature, soma size, in addition to cell process length and number

209 (Karperien et al., 2013) were measured live at 40x for every cell within a $23672.24 \mu \mathrm{m}^{2}$ region of

210 interest (ROI), with 3 ROIs each for the dorsal and ventral hippocampus. Further, no more than one ROI

211 was taken from an individual tissue slice, and ROIs were defined in 3 consistent locations in the GCL

212 for both the dorsal and ventral hippocampus within each animal. Cells were defined by the presence of

213 an Iba-1-IR cell body within the ROI, and this definition did not necessitate the presence of cell

214 processes, by that ensuring the inclusion of any cells with amoeboid morphology. The average process

215 length per cell was calculated using the total length and number of processes for each cell, and

216 subsequently an average process length was calculated for each animal. Both primary (extending

217 directly from the cell body) and secondary processes were taken into account in the analyses. There

218 were no significant differences between groups in the number of Iba-1-IR cells that fell within the ROIs

219 and were used for morphological analyses (see Table 1). 
220 In an additional analysis of microglial morphology, Iba-1-IR cells were categorized into one of three

221 morphological phenotypes, adapted from other methods (Haim et al., 2017; Schwarz et al., 2012).

222 Specifically, as depicted in the representative photomicrographs in Fig. 6D-F, cells were categorized as

223 1) ramified microglia (highly branched, with longer processes), stout microglia (few, shorter processes),

224 or 3) amoeboid microglia (no processes). This was performed under a 40X objective, in 4 hippocampal

225 slices per animal, and involved a classification of all Iba-1-IR cells in the GCL, SGZ, and an

226 approximately $50 \mu \mathrm{m}$ band of the ML. For each animal, the percentage of cells of each morphological

227 phenotype was calculated.

228 2.5.2. DCX: Under a 100x objective on an Olympus CX22LED brightfield microscope, DCX-IR cells in 229 the granule cell layer (GCL) were exhaustively counted in every $10^{\text {th }}$ section of the hippocampus along 230 the rostral-caudal axis. Thus, raw counts were multiplied by a factor of 10 to obtain an estimate of the 231 total number of DCX-IR cells in the hippocampus. Previous work indicates that certain conditions or 232 experiences, including reproductive experience (Workman et al., 2015), can alter the rate at which newly 233 produced hippocampal neurons mature (Overstreet-Wadiche et al., 2006), and this can have functional 234 consequences as changes in the maturational timeline can alter the rate of functional integration of these new neurons into existing hippocampal networks. Thus, to examine whether pregnancy and motherhood can alter the rate at which newly produced hippocampal neurons mature, we investigated the maturational stages of DCX-immunoreactive cells. Specifically, using the $100 \times$ objective on an Olympus CX22LED brightfield microscope, 50 DCX-IR cells (25 dorsal GCL and 25 ventral GCL; each taken from 3 slices) were randomly selected for each animal for maturational staging. Cells were

240 categorized into one of three maturational stages, based on previously established criteria (Plümpe et al.,

241 2006): 1) proliferative (no process or short process), 2) intermediate (medium process with no

242 branching), or 3) post-mitotic (long processes with branching in the GCL and ML). DCX-

243 immunolabeled sections were also utilized to measure GCL areas in every $10^{\text {th }}$ section of the

244 hippocampus, using images taken at 4x and the ImageJ software. Volume was estimated using these 245 areas and following Cavalieri’s principle.

246 2.5.3. Ki67: Under a 100x objective on an Olympus CX22LED microscope equipped with 247 epifluorescence, Ki67-IR cells in the GCL and SGZ of the DG were exhaustively counted in every $10^{\text {th }}$ 248 section of the hippocampus along the rostral-caudal axis. Raw counts were multiplied by a factor of 10 249 to obtain an estimate of the total number of Ki67-IR cells in the DG.

\section{$250 \quad$ 2.6. Serum cytokine and hormone quantification}


251 A multiplex immunoassay kit (V-PLEX Proinflammatory Panel 2, Rat) was purchased from Meso-Scale

252 Discovery (Rockville, MD) and used according to manufacturer instructions to measure serum cytokine

253 levels. The antibody pre-coated plates allowed for the simultaneous quantification of the following

254 cytokines: Interferon-gamma (IFN- $\gamma$ ), Interleukin-1beta (IL-1 $\beta$ ), Interleukin-4 (IL-4), Interleukin-5 (IL-

255 5), Interleukin-6 (IL-6), chemokine (C-X-C motif) ligand 1 (CXCL1), Interleukin-10 (IL-10),

256 Interleukin-13 (IL-13), and tumor necrosis factor alpha (TNF- $\alpha$ ). Samples were run in duplicates, and

257 plates were read with a Sector Imager 2400 (Meso Scale Discovery), and data was analyzed using the

258 Discovery Workbench 4.0 software (Meso Scale Discovery). The assays’ lower limits of detection

259 (LLOD), which varied between analytes and plates (2 plates total), were as follows (pg/mL): IFN- $\mathrm{\gamma}$ :

260 0.163-0.266; IL-10: 0.233-0.313; IL-13: 0.78-2.7; IL-1ß:1.48-1.62; IL-4:0.179-0.298; IL-5: 7.64-9.8;

261 IL-6: 2.48-2.49; CXCL1: 0.085-0.164; and TNF- $\alpha$ : 0.156-0.186. Any values below the LLOD were

262 assigned $0 \mathrm{pg} / \mathrm{mL}$, as we have done previously (Bodnar et al., 2017). All samples were within the

263 detection range for TNF- $\alpha$, CXCL1, and IL-10. One sample fell below the LLOD for each of IFN- $\gamma$, IL-

264 4, and IL-13. For three cytokines, a number of samples fell below the LLOD ( $\mathrm{n}=12$ for IL-6, n=17 for

265 IL-1 $\beta$, and n=36 for IL-5). This panel was chosen as it includes a broad range of cytokines, some

266 traditionally considered proinflammatory (IL-1 $\beta$, IFN-y, TNF- $\alpha$ ), anti-inflammatory (IL-4, IL-10), and

267 pleiotropic (IL-6), in addition to the chemokine CXCL1 which is important for neurotrophil recruitment.

268 Therefore, combined, these markers provide a comprehensive view of the inflammatory milieu.

269 Steroids hormones modulate neuroplasticity (Mahmoud et al., 2016b) and interact with the immune

270 system (Schumacher et al., 2014). Further, circulating concentrations increase dramatically during

271 pregnancy, drop abruptly at parturition, and diminish in ageing females. To investigate the potential

272 mediating roles of steroid hormones, we measured $17 \beta$-estradiol, the most potent of the estrogens and

273 the most abundant in premenopausal women and younger female rats (Rannevik et al., 1986). Serum

$274 \quad 17 \beta$-estradiol concentrations were quantified in sample taken at perfusion, using an ultra-sensitive

275 estradiol radioimmunoassay (Beckman Coulter, Prague, Czech Republic) and in accordance with

276 manufacturer's instructions, with all samples run in duplicates. The assay sensitivity is $2.2 \mathrm{pg} / \mathrm{ml}$, and the

277 average inter- and intra-assay coefficients of variation were $<15 \%$.

\subsection{Statistical analyses}

279 Statistical analyses were performed using Statistica software (Tulsa, OK). All dependent variables were

280 subjected to the Kolmogorov-Smirnov test for normality, and Bartlett's test for heterogeneity of

281 variance. TNF- $\alpha$, IL-1 $\beta$, IL-5, and IL-6 data were not normally distributed and therefore Box-Cox 
282 transformed prior to analyses. There were few violations to the heterogeneity of variance assumption,

283 however these were corrected after Box-Cox transformation. Neural measures (DCX- and Ki67-IR cell 284 number, Iba-1-IR density, length and number of processes) and serum measures (17 $\beta$-estradiol, IFN- $\gamma$, 285 IL-1 $\beta$, IL-4, IL-5, IL-6, CXCL1, IL-10, IL-13, and TNF- $\alpha$ ) were each analyzed using factorial analysis 286 of variance (ANOVA), with time (GD13/7 mo., PPD8/7.5 mo., PPD30/8 mo., PPD90/10 mo., 287 PPD180/13 mo.) and reproductive status (primi-gravid/parous, nulliparous) as the between-subject 288 factors. Post-hoc analyses utilized Fisher's LSD. A priori we expected parity to modulate the age-related 289 changes in cytokine levels, and density/morphology of Iba-1-IR cells. Any a priori comparisons were 290 subjected to a Bonferroni correction. Pearson's correlations were performed on dependent variables of 291 interest, and Fisher's z-transformation of correlation coefficients was used to assess the significance of 292 the difference between correlations in nulliparous and primiparous groups. Serum 17 $\beta$-estradiol 293 concentrations were used as a covariate in ANOVAs, as circulating concentrations can influence 294 neuroplastic and immune measures (Mahmoud et al., 2016b; Schumacher et al., 2014); ANOVAs are 295 reported with the covariate in instances where there was a significant main effect of the covariate. 296 Finally, as an exploratory approach, and to complement our findings from ANOVA analyses, we ran a 297 principal component analysis (PCA) on the cytokine data, with the purpose of deriving information 298 about the amount of variance accounted for by potential cytokine networks within the dataset.

\section{3. Results}

\subsection{Serum 17 $\beta$-estradiol was reduced in the early postpartum and increased in the late postpartum}

301 As expected, 17 $\beta$-estradiol was reduced in the early postpartum period at PPD8, relative to age-matched 302 nulliparous rats ( $p=0.015$; reproductive status by time interaction: $F(4,36)=4.45, p=0.005$; Fig. 2). Further, 303 primiparous rats at PPD30 had higher serum 17 $\beta$-estradiol concentrations than age-matched nulliparous 304 rats ( $<<0.008$; Fig. 2). Finally, 17 $\beta$-estradiol concentrations at PPD30 and PPD90 were significantly higher 305 than all other primiparous groups (p’s < 0.03), but there were no significant differences between any of 306 the nulliparous groups (all p’s $>0.19)$. There was also a significant main effect of time $(\mathrm{p}<0.01)$ but not of 307 reproductive status $(\mathrm{p}=0.41)$. 


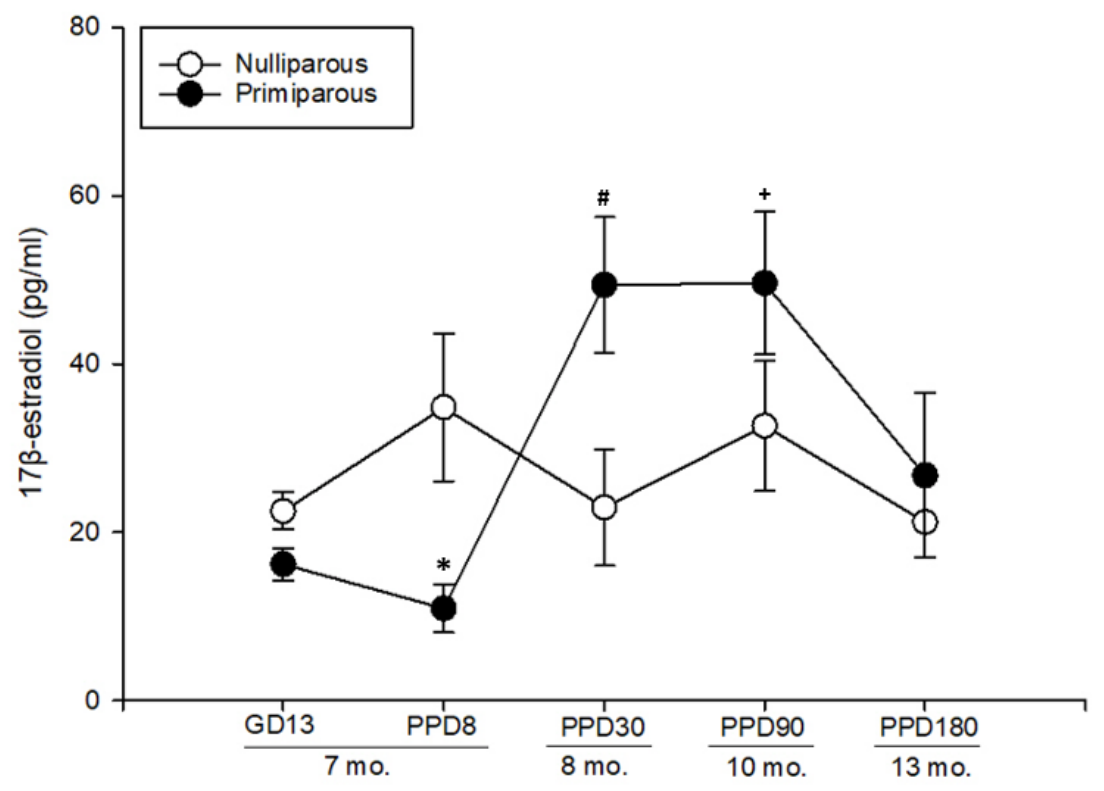

Figure 2. Serum concentrations of $17 \beta$-estradiol at perfusion in primiparous and nulliparous rats. The $x-$ axis represents time relative to gestation and parturition in primiparous rats, and approximate age in months. $17 \beta$-estradiol was significantly reduced in the early postpartum period at PPD8, but significantly increased in the late postpartum period at PPD30 and PPD90. * indicates p $=0.015$, significantly lower than age-matched nulliparous rats; \# indicates $\mathrm{p}<0.03$, significantly higher that 8-month-old nulliparous rats, and primigravid/parous rats at GD13, PPD8, and PPD180; + indicates $\mathrm{p}<0.03$, significantly higher than primigravid/parous rats at GD13, PPD8, and PPD180. Data are represented in mean values \pm SEM. $\mathrm{GD}=$ gestation day, $\mathrm{PPD}=$ postpartum day.

\subsection{Parity and age had no significant effect on granule cell layer volume}

Granule cell layer volume was not significantly affected by age, parity, or age by parity interaction (all p’s $>0.48$ ), thus all further analyses were performed on the number, rather than density, of Ki67- and DCX-IR cells. postpartum period, and declined in middle-age in nulliparous rats only

314 The number of DCX-IR cells was significantly reduced in primi-gravid and -parous rats relative to age315 matched nulliparous controls at GD13, PPD8, and PPD30 (p’s <0.001; significant time by reproductive 316 status interaction; $F(4,38)=6.7213$, $p<0.001$; Fig. 3). There were also significant main effects of time 317 and reproductive status (all p’s < 0.001). 
320 nulliparous age groups (all p’s<0.014), with the exception of a non-significant decline from 10 to 13

321 months ( $p=0.11$; significant time by reproductive status interaction; $F(4,38)=6.7213, p<0.001$; Fig. 3).

322 There was also a significant decline in DCX-IR cell number in primiparous rats at PPD30 relative to

323 GD13 and PPD8 (p’s<0.017; Fig. 3). However, the trajectory of age-related change in DCX-IR cell

324 number was significantly altered by parity in middle-age; unlike nulliparous rats, primiparous groups

325 had no significant difference in the number of DCX-IR cells from 8 to 10 months of age $(p=0.10)$. Based

326 on previous findings (Barha et al., 2015; Galea et al., 2018), we expected parity to increase neurogenesis

327 in middle age. Indeed, we found a significant increase in DCX-IR cells from 8 to 13 months of age in

328 primiparous rats ( $\mathrm{p}=0.044$; one-tailed).

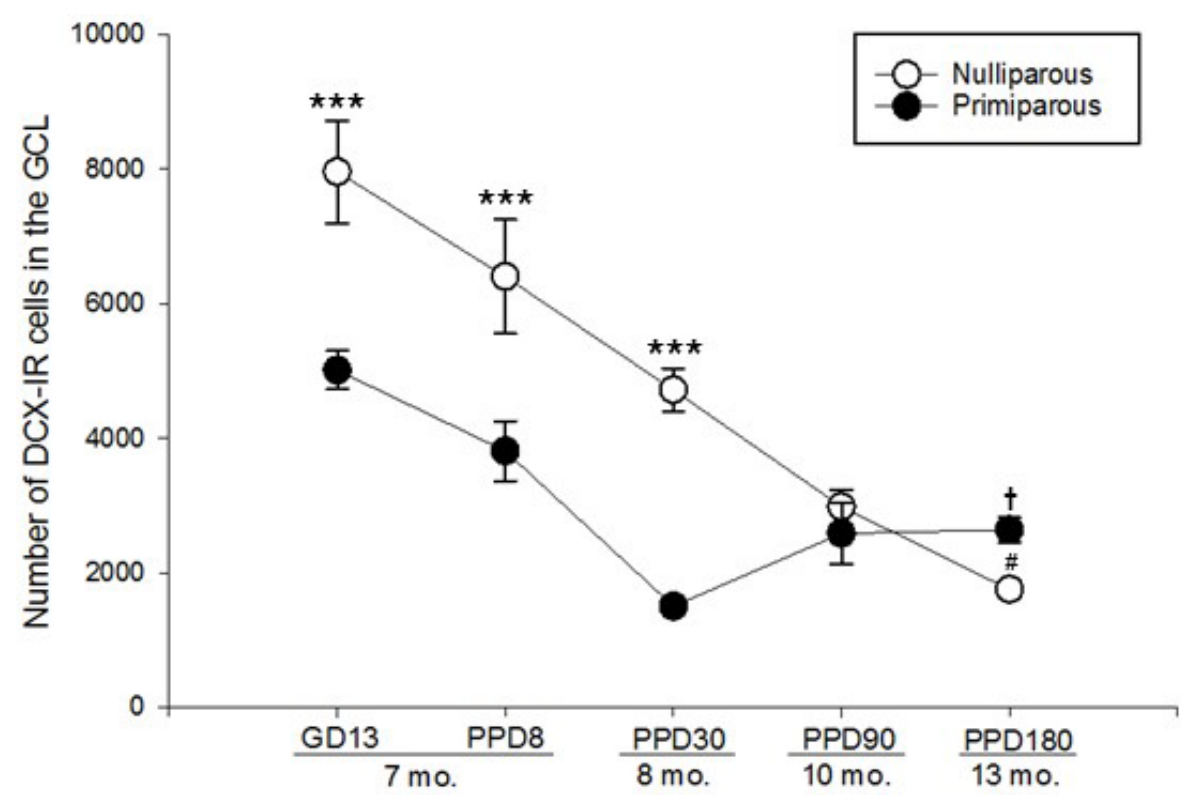

Figure 3. Estimated total number of doublecortin (DCX)-immunoreactive (IR) cells in the granule cell layer of primiparous and nulliparous rats across 7-13 months of age. The x-axis represents time relative to gestation and parturition in primiparous groups, and approximate age in months. DCX-IR cell number was significantly reduced in primiparous rats in mid-gestation and until postpartum day 30. Between 8 and 13 months of age, DCX-IR cell number significantly declined in nulliparous rats, but significantly increased in primiparous rats. Data are represented in mean values \pm standard error of the mean $(\mathrm{SEM}) . \mathrm{GCL}=$ granule cell layer, DCX-IR= doublecortinimmunoreactive, $\mathrm{GD}=$ Gestations Day, $\mathrm{PPD}=$ Postpartum Day, mo.= approximate age in months. $* * *$ indicates $\mathrm{p}<0.001$, significantly different from age-matched primiparous group. \# denotes $\mathrm{p}<0.001$, significantly different from 8-month-old nulliparous group. † indicates $\mathrm{p}=0.044$, significantly different from primiparous rats at PPD30.

\subsection{Parity and age had no significant effect on the maturational stage of DCX-IR cells}

330 Regardless of age and reproductive status, the percentage of proliferative DCX-IR cells was

331 significantly higher than that of intermediate DCX-IR cells $(\mathrm{p}<0.001)$, and the percentage of post-mitotic

332 DCX-IR cells was significantly higher than that of intermediate and proliferative DCX-IR cells

333 (p’s<0.001; significant main effect of DCX maturational stage, F(2, 76)=107.17, p<0.001; Table 2). 
334 There were no other significant main effects, and no significant interactions (all p’s $>0.14$ ).

\section{3.5. Ki67-IR cells declined in mid-gestion and the early postpartum period in primiparous rats,} 336 and with age regardless of reproductive status

337 Parity significantly reduced the number of Ki67-IR cells in the GCL $(F(1,38)=9.2681$, $p=0.004$;

338 significant main effect of reproductive status; Fig. 4A). Regardless of reproductive status, Ki67-IR cells 339 declined significantly with age $(F(4,38)=16.505$, $p<0.001$; main effect of time; Fig. 4A), in which there 340 was a significant difference between all age groups (p’s < 0.005), with the exception of non-significant 341 differences from 7 to 7.5 months, 8 to 10 months, and 10 to 13 months (p’s $>0.05$ ). Although there was

342 no significant age by parity interaction $(\mathrm{p}=0.13)$, a priori we expected a decline in cell proliferation in

343 the early postpartum period based on previous work (Leuner et al., 2007). Planned comparisons revealed 344 a significant decline in Ki67-IR cells on PPD8 ( $\mathrm{p}=0.013$; one-tailed), in addition to a significant decline 345 on GD13 ( $p=0.004)$, relative to age-matched nulliparous controls in both instances (Fig. 4A).
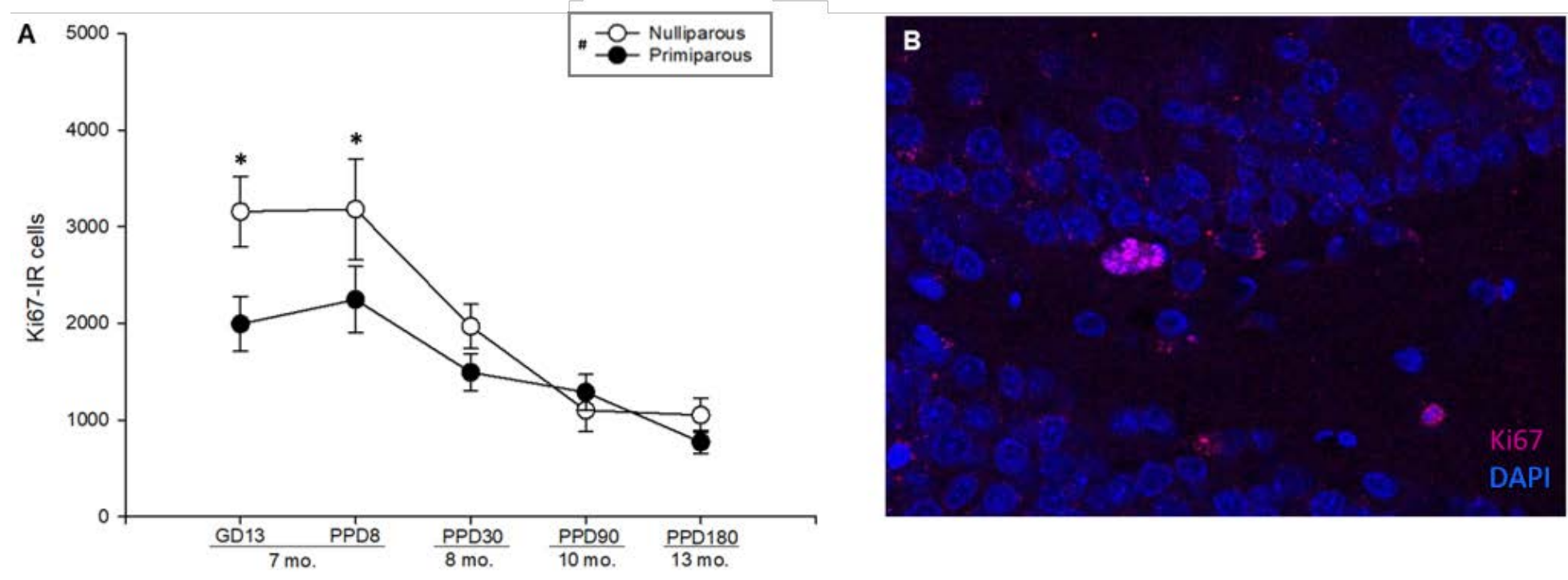

Figure 4. (A) Estimated total number of Ki67-immunoreactive (IR) cells in the granule cell layer and subgranular zone of primiparous and nulliparous rats across 7-13 months of age. The $\mathrm{x}$-axis represents time relative to gestation and parturition in primiparous groups, and approximate age in months. Ki67-IR cell number was significantly reduced in primiparous rats in mid-gestation and the early postpartum period, and declined with age regardless of reproductive status. Data are represented in mean values \pm standard error of the mean (SEM). Ki67IR = Ki67-immunoreactive, GD= Gestations Day, PPD= Postpartum Day, mo.= approximate age in months. * indicates $\mathrm{p}<0.014$, significantly different from age-matched primiparous group. \# denotes $\mathrm{p}=0.004$, significant main effect of reproductive status. (B) Representative photomicrograph of the dentate gyrus, showing Ki67-IR cells (pink), counterstained with DAPI (blue). 
3.6. Density of Iba-1-IR cells increased in the late postpartum period and fluctuated significantly with age in primiparous but not in nulliparous rats

348 Because we quantified Iba-1-IR cells in 4 sections per animal, the density of IR cells was analyzed, as 349 we have done previously (Mahmoud et al., 2016a). Age significantly affected the density of Iba-1-IR 350 cells in the dentate gyrus, with higher density at 8 and 13 months relative to 7.5 and 10 months of age 351 (p’s<0.02; significant main effect of time, $F(4,39)=3.43$, p=0.017). We expected alterations in the 352 density of Iba-1-IR cells in the postpartum period due to previous findings (Haim et al., 2017). A priori 353 comparisons show that the density of Iba-1-IR cells fluctuated significantly across time in primiparous 354 rats; density was increased in the late postpartum period at PPD30 relative to PPD8 (p=0.002), and in middle-aged rats at PPD180 relative to PPD8 ( $<<0.003$; Fig. 5A). On the other hand, density did not significantly change across age in nulliparous rats (all p’s >0.17; Fig. 5A). There were no significant differences between nulliparous and primiparous groups across age (all p’s $>0.3$ ), except for a trend for higher density at PPD30 relative to age-matched nulliparous controls ( $p=0.09$; Fig. 5A). There was no significant main effect of reproductive status and no significant interaction (p’s>0.28).

\subsection{Average length and number of Iba-1-IR cell processes changed significantly with age and} reproductive status.

Iba-1-IR cells displayed significantly shorter processes in the early postpartum period at PPD8 relative to nulliparous controls ( $\mathrm{p}=0.005$; Fig. 5B), and to primiparous rats at GD13 $(\mathrm{p}<0.002)$. There was a decline in average length of Iba-1-IR cell processes at 10 months of age, relative to 7 months in primiparous rats, and to 7, 7.5, and 8 months in nulliparous rats (all p's $<0.027$; significant time by reproductive status interaction $\mathrm{F}(4,38)=4.67$, p=0.004; Fig. 5B). However, there was no further significant change in average process length between 10 and 13 months of age, regardless of reproductive status ( $\mathrm{p}$ ’s $>0.23$ ). There was also a significant main effect of the covariate serum $17 \beta$ estradiol ( $\mathrm{p}=0.012)$, a trend towards a significant main effect of time ( $\mathrm{p}=0.067)$, but no significant main effect of reproductive status $(\mathrm{p}=0.42)$.

371 The average number of Iba-1-IR cell processes increased significantly with age in nulliparous rats, 372 where significantly more processes were found in 13- compared to 8-month-old rats ( $<<0.002)$, but 373 missed significance compared to 7.5-month-old rats ( $\mathrm{p}=0.027$; planned comparisons; Fig. 5C). On the 374 other hand, there were no significant differences in the average number of cell processes between 375 primiparous rats across age (all p’s $>0.06$ ). There were trends towards significant main effects of time 
$376(\mathrm{p}=0.054)$ and reproductive status $(\mathrm{p}=0.056)$ but no significant time by reproductive status interaction

$377 \quad(\mathrm{p}=0.11)$.

378 There was a trend for primiparity to decreased the soma size of Iba-1-IR cells relative to nulliparity (F(1, $37938)=5.2646$, p=0.092; main effect of reproductive status; Fig. 5D). While there was no significant age by 380 parity interaction ( $\mathrm{p}=0.80$ ), the effects of primiparity to reduce soma size appears to be driven by the 381 PPD30, 90, and 180 groups. In addition, there was a significant main effect of time $(\mathrm{F}(4,38)=5.28$, $382 \mathrm{p}<0.001)$.

A

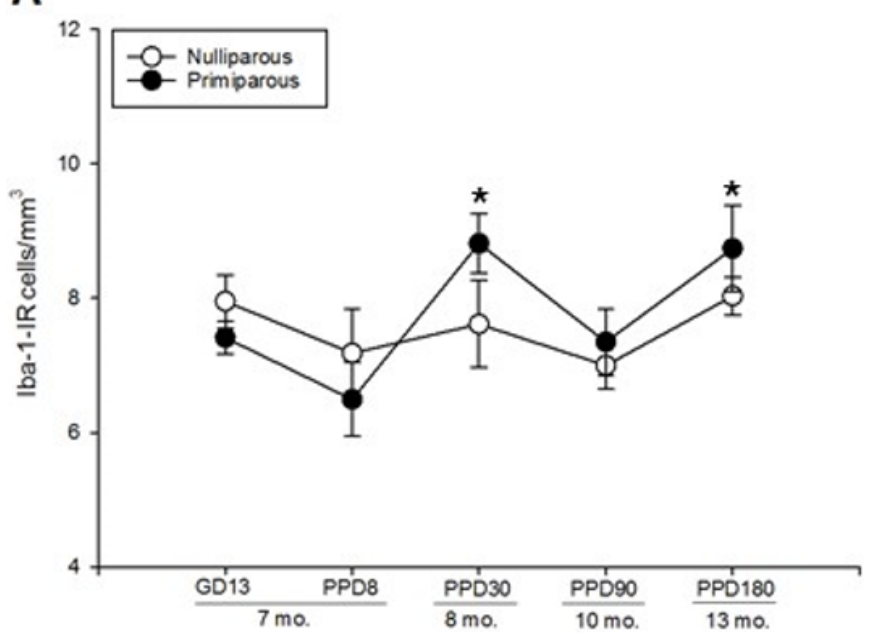

C

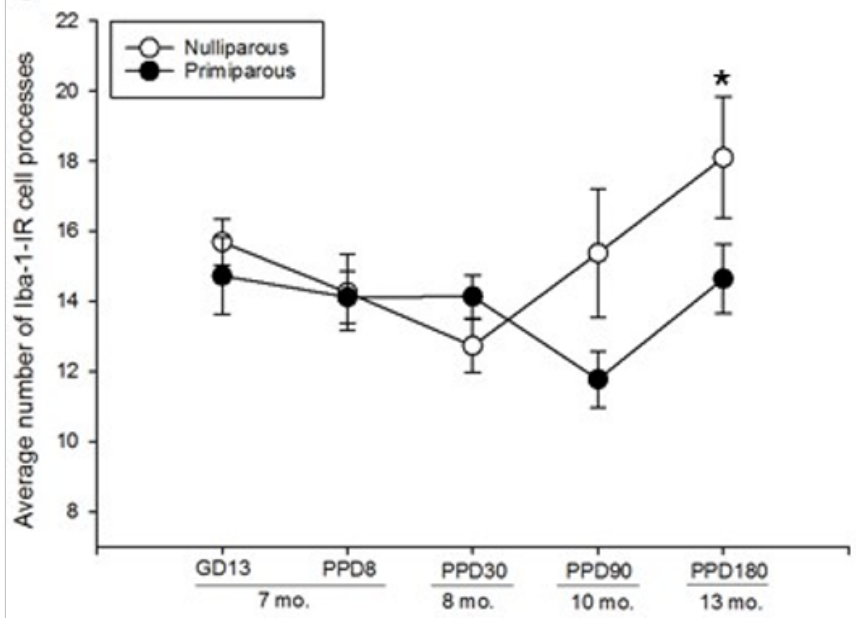

B

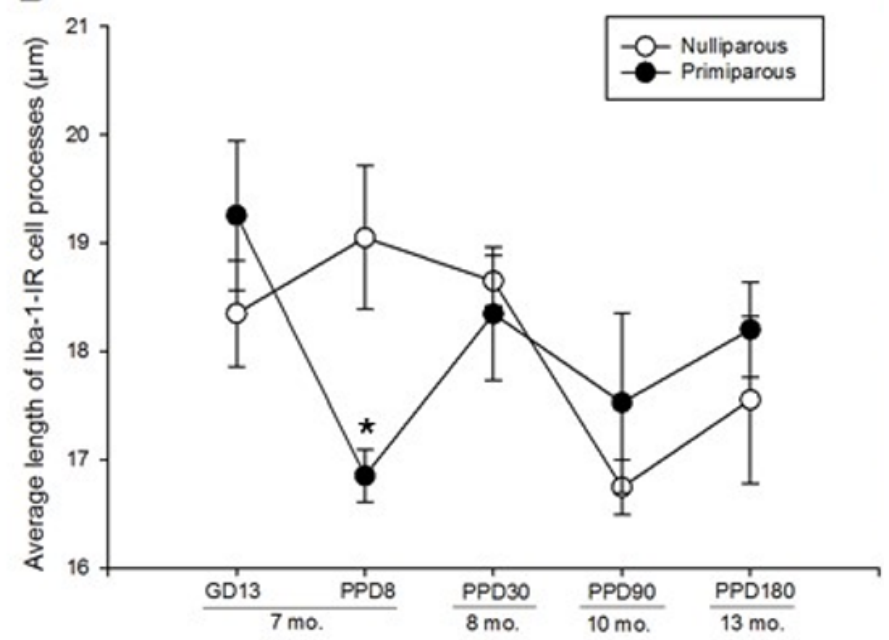

D

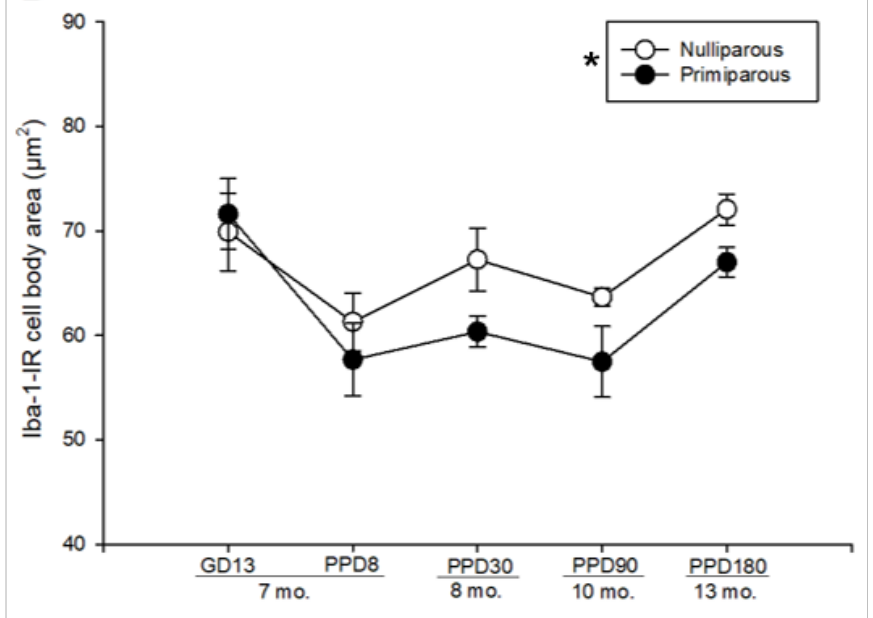

Figure 5. Density and morphology of Iba-1-immunoreactive cells in the dentate gyrus of primiparous and nulliparous rats. The $\mathrm{x}$-axis represents time relative to gestation and parturition in primiparous rats, and approximate age in months. (A) Iba-1-IR cell density was stable across age in nulliparous rats, but fluctuated significantly in primiparous rats, with increased density at PPD30 and PPD180, relative to PPD8. * indicates $\mathrm{p}<0.003$, significantly different from primiparous group at PPD8. (B) Average length of Iba-1-immunoreactive cell processes was reduced at PPD8, and decreased with age regardless of reproductive status. * denotes $\mathrm{p}<0.006$, significantly different from age-matched nulliparous controls, and from primiparous group at GD13 (C) Average number of Iba-1-immunoreactive cell processes increased significantly with age in nulliparous but not primiparous rats. * indicates $\mathrm{p}<0.002$, significantly different from 8-month-old nulliparous group. (D) There was a trend towards significance for parity to was reduced soma area of Iba-1-IR cells * indicates $\mathrm{p}=0.092$, main effect of reproductive status. Data are represented in mean values $\pm \mathrm{SEM}$. GD= gestation day, PPD= postpartum day, mo.= age in months, Iba-1-IR= Iba-1-immunoreactive. 


\subsection{The percentage of Iba-1-IR cells of stout morphology increased, and that of ramified}

\section{morphology decreased, in the early postpartum period}

There was a significant Iba-1 morphology by time by reproductive status interaction $(F(8,74)=2.066$,

$\mathrm{p}=0.05$; Fig.6), in which the percentage of ramified Iba-1-IR cells was reduced in primiparous rats at

PPD8 relative to age-matched nulliparous controls, primigravid rats at GD13, and primiparous rats at

increased in primiparous rats at PPD8 relative to age-matched nulliparous controls and primigravid rats

392 Iba-1 morphology ( $<<0.001$ ), a trend for a significant Iba-1 morphology by time interactions ( $\mathrm{p}=0.087$ ),

393 but no other significant main effects or interactions (p's $>0.49$ ).
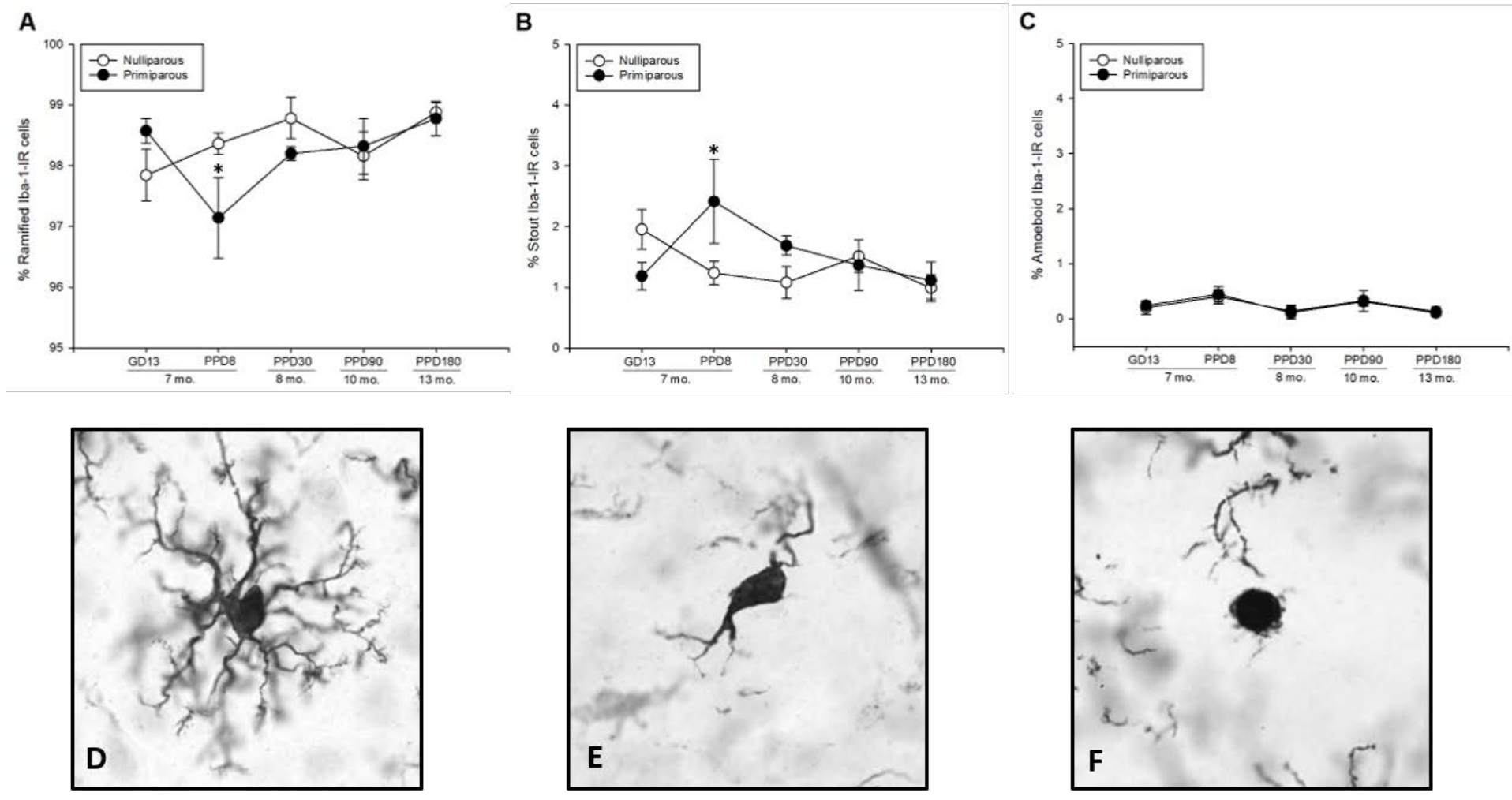

Figure 6. Percentage of Iba-1-immunoreactive cells of ramified (A), stout (B), and amoeboid (C) morphology in the dentate gyrus. The $\mathrm{x}$-axis represents time relative to gestation and parturition in primiparous rats, and approximate age in months. (A) the percentage of ramified Iba-1-IR cells was transiently reduced in the early postpartum period. * indicates $\mathrm{p}<0.02$, PPD8 significantly lower than 7.5-month-old nulliparous rats, primigravid rats at GD13, and primiparous rats at PPD30. (B) the percentage of stout Iba-1-IR cells was transiently increased in the early postpartum period. * indicates $\mathrm{p}<0.007$, PPD8 significantly higher than 7.5 -month-old nulliparous rats and primigravid rats at GD13. (C) the percentage of amoeboid Iba-1-IR cells did not differ between groups. Representative photomicrographs of Iba-1-IR cells of ramified (D), stout (E) and amoeboid (F) morphology captured at 60x. Data are represented in mean values \pm SEM. GD= gestation day, $\mathrm{PPD}=$ postpartum day, mo.= age in months, Iba-1-IR= Iba-1-immunoreactive. 

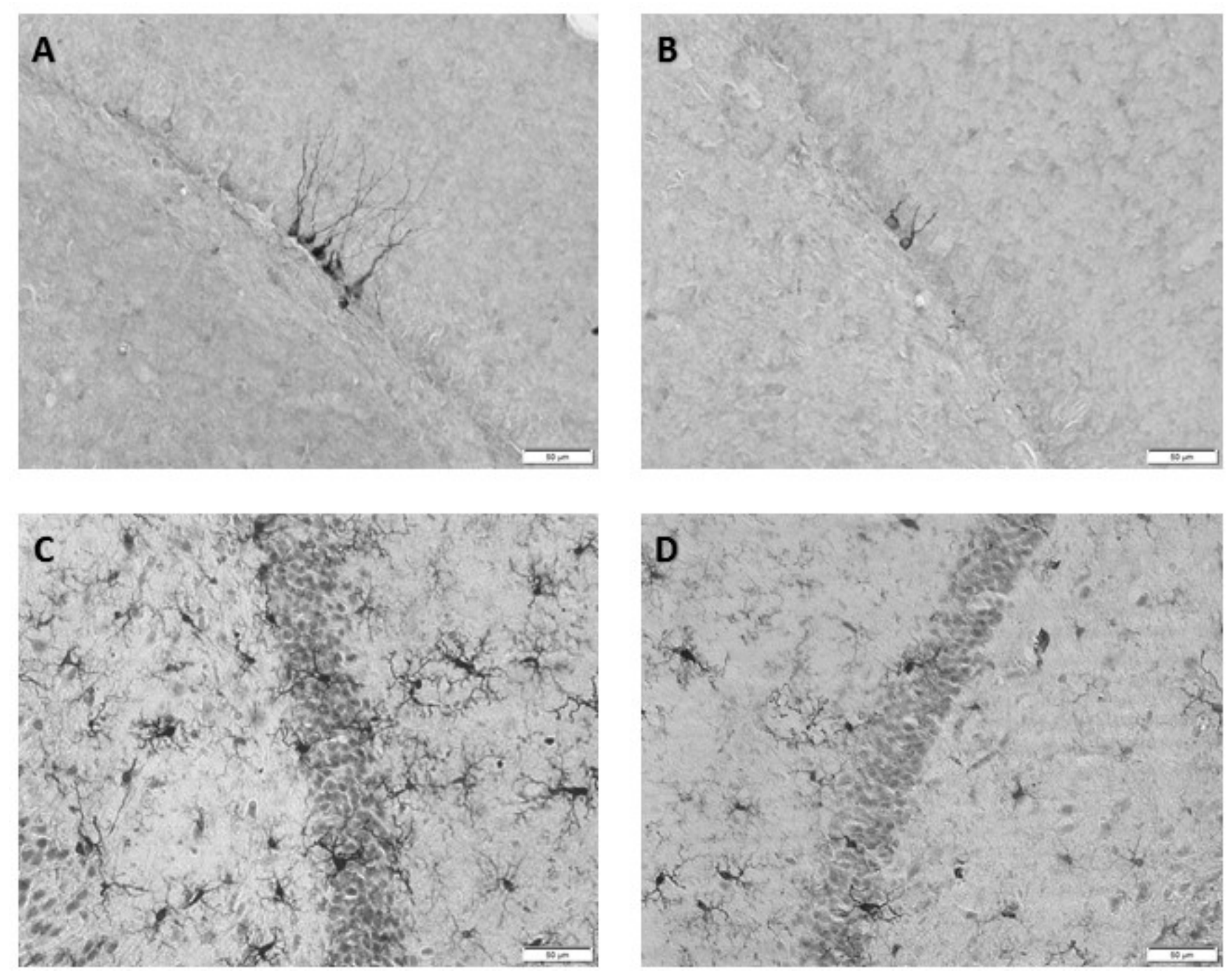

Figure 7. Representative photomicrographs of the granule cell layer in the dentate gyrus. (A) Doublecortin-immunoreactive cells in an 8-month-old nulliparous rat. (B) Doublecortin-immunoreactive cells in an 8-month-old primiparous rat on postpartum day 30. (C) Iba-1-immunoreactive cells in a 7.5month-old nulliparous rat. (D) Iba-1-immunoreactive cells in a 7.5-month-old primiparous rat on postpartum day 8.

\subsection{Serum IFN- $\gamma$ and IL-10 showed an age-related increase in nulliparous but not primiparous}

395 rats.

396 There was a significant ageing-related increase in IFN- $\gamma$ in nulliparous rats, in which 13-month-old

397 nulliparous rats had significantly higher IFN- $\gamma$ levels than all other nulliparous groups (all p’s <0.004;

398 planned comparisons; Fig. 8A). No significant differences were found in IFN- $\gamma$ levels between any 399 primiparous groups (all p’s $>0.04$, non-significant due to Bonferroni correction). Further, at 13 months,

$400 \quad$ IFN- $\gamma$ levels were higher in nulliparous relative to primiparous rats $(p=0.023$; Fig. 8A). There was also

401 a significant main effect of time $(\mathrm{p}<0.001)$, but not reproductive status $(\mathrm{p}=0.14)$ nor an interaction

$402(\mathrm{p}=0.325)$. 
Similarly, serum IL-10 increased significantly with age in nulliparous but not primiparous rats;

404 significantly higher levels of IL-10 were detected in 13-month old nulliparous rats relative to all other

405 nulliparous groups $(\mathrm{P}<0.002$; Fig. 8B), but no significant differences were found between any of the

406 primiparous groups (all p’s>0.05; planned comparisons; Fig. 8B). There was also a significant main

407 effect of time $(\mathrm{p}<0.003)$, but not reproductive status $(\mathrm{p}=0.11)$ nor an interaction $(\mathrm{p}=0.45)$.

A

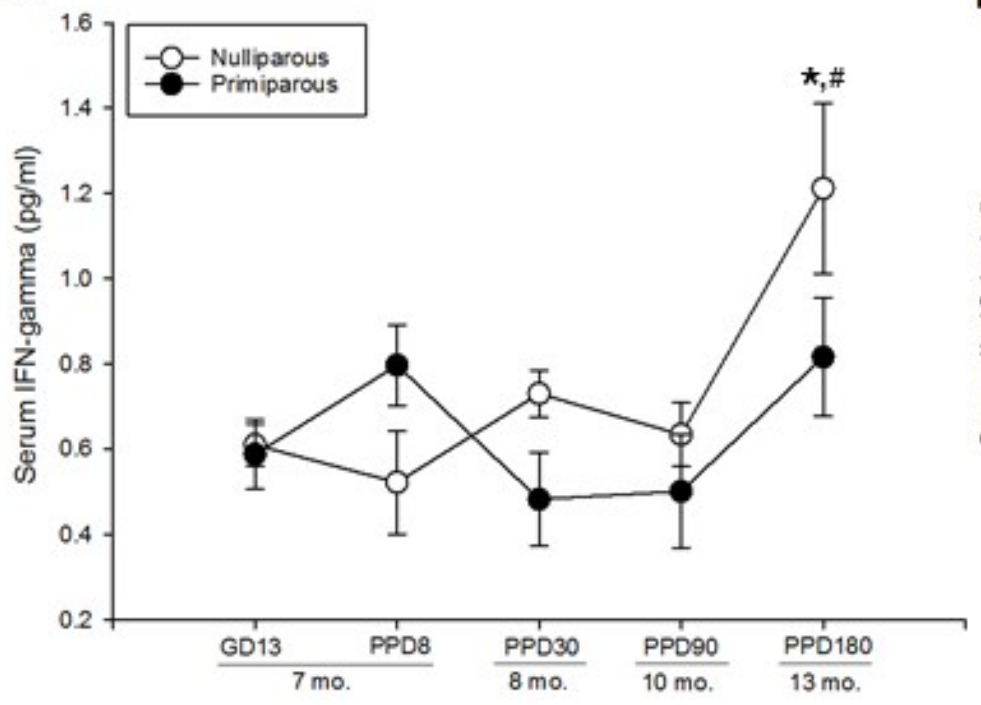

B

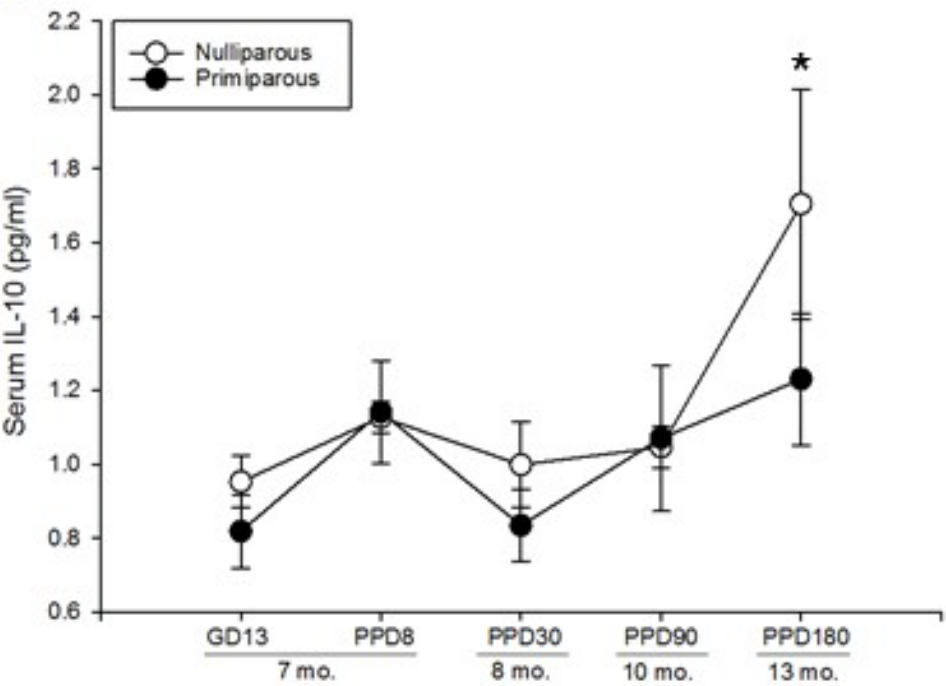

Figure 8. Serum levels of interferon- $\boldsymbol{\gamma}$ (A) and interleukin-10 (B) in primiparous and nulliparous rats. The x-axis represents time relative to gestation and parturition in primiparous groups, and approximate age in months. There was a significant ageing-related increase in IFN- $\gamma$ (A) and IL-10 (B) in nulliparous but not primiparous rats. * indicates $\mathrm{p}<0.004$, significantly different from all other nulliparous groups. \# indicates $\mathrm{p}=0.023$, significantly different from13-month-old primiparous rats. Data are represented in mean values \pm SEM. IFN- $\gamma=$ interferon gamma, IL-10= interleukin $10, \mathrm{GD}=$ gestation day, $\mathrm{PPD}=$ postpartum day.

3.10. Serum IL-4 was transiently increased in the early postpartum then persistently reduced by parity

Nulliparous rats had higher levels of IL-4 than primiparous rats, regardless of time point (main effect of reproductive status: $F(1,38)=7.63$, $p<0.009$, Fig. 9A). Regardless of reproductive status, there was an age-related increase in serum IL-4, with significantly elevated levels at 13 months compared to all groups at 8 months of age and younger (all p's $<0.04$; main effect of time: $F(4,38)=4.29, p<0.006$ ).

414 There was no significant reproductive status by time interaction $(\mathrm{F}(4,38)=1.73$, $\mathrm{p}=0.16)$, but a priori we 415 expected cytokine levels to be altered in the early postpartum period in primiparous rats. Indeed, 416 primiparous rats showed a trend for a transient increase in serum IL-4 in the early postpartum period, 417 with higher levels at PPD8 relative to GD13 ( $\mathrm{p}=0.029)$ and PPD30 ( $\mathrm{p}=0.027$; a priori comparisons, 418 missing significance with correction; Fig. 9A). In age-matched nulliparous control groups, serum IL-4 419 was not significantly different in 7.5- relative to 7-or 8-month-old rats (p’s >0.45). 


\subsection{Serum IL-5 was transiently reduced during gestation and showed an age-related decline in} nulliparous but not primiparous rats.

422 Serum IL-5 levels were significantly reduced in gestation (GD13), relative to age matched nulliparous

423 controls ( $p=0.006$; time by reproductive status interaction, $F(4,37)=3.31 ., p=0.020$; Fig. 9B). Further,

424 IL-5 levels declined with age in nulliparous animals, as higher levels were detected at 7 months relative 425 to 8, 10 and 13 months (all p’s<0.02; Fig. 9B). Although non-significant, IL-5 levels increased in 426 primiparous animals with age, suggesting a reversed pattern of age-related changes in IL-5 compared to 427 nulliparous rats. There were no significant main effects of time or reproductive status (all p’s $>0.1$ ).

A

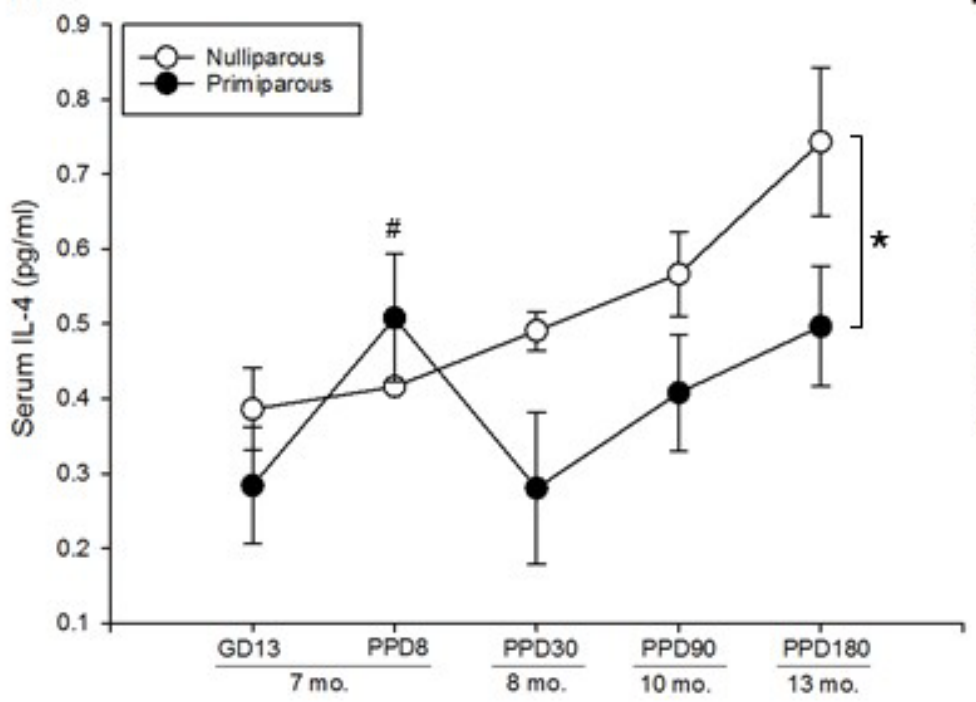

B

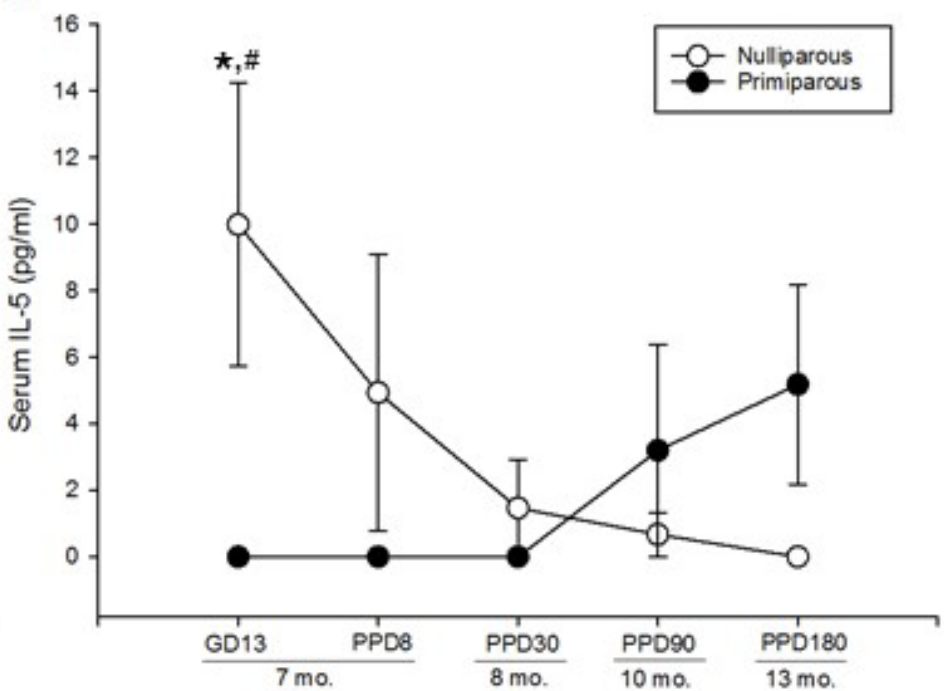

Figure 9. Serum levels of interleukin-4 (A), and interleukin-5 (B) in primiparous and nulliparous rats. The x-axis represents time relative to gestation and parturition in primiparous groups, and approximate age in months. (A) Serum IL-4 levels were transiently increased in primiparous rats at PPD8, but persistently suppressed by parity thereafter. \# indicates $\mathrm{p}<0.03$, trend towards significance relative to GD13 and PPD30. * indicates $\mathrm{p}<0.009$, main effect of primiparity to reduce IL-4 levels (B) Serum IL-5 was transiently blunted during gestation, and a significant age-related decline in serum IL-5 was found only in nulliparous rats. * indicates p=0.006, significantly higher than primigravid rats at GD13. \# indicates $\mathrm{p}<0.02$, significantly higher than all nulliparous groups between 8 and 13 months of age.

429 Regardless of reproductive status, there was a trend towards significance for a main effect of time to

430 affect IL-13 $(F(4,37)=2.46, p=0.06$; Fig. 10A), where IL-13 levels were elevated at 13 months

431 compared to 8 months ( $\mathrm{p}<0.002$; planned comparisons). There were no significant main effects of 
432 reproductive status or $17 \beta$-estradiol (covariate), and no time by reproductive status interaction (all p’s

$433>0.4)$. There were no significant main effects of reproductive status or age, nor an interaction for serum

434 concentrations of IL-6 (all p’s > 0.21, Fig. 10B), CXCL1 (all p’s >0.40 Fig. 10C), or IL-1 $\beta$ (all p’s >

435 0.32, Fig. 10D). There were trends towards significance for a main effect of parity to increase TNF- $\alpha$ (p

$436=0.064)$ and a main effect of time $(\mathrm{p}=0.061)$ for TNF- $\alpha$ to decline with age, but no significant

437 reproductive status by time interaction $(p=0.72$. Fig. 10E).

\subsection{Principal Component Analysis of serum cytokines}

439 The model generated 4 principal components, which accounted for $82.6 \%$ of the variance within the 440 dataset, with the first principal component explaining $40.4 \%$ of the variance, the second $18.2 \%$, the third 441 12.6\%, and the fourth 11.4\%. Interestingly, IFN- $\gamma$, IL-10, IL-13, and IL-4 loaded heavily onto Principal

442 Component 1 (PC1; see Table 3). These same cytokines also showed the most robust alterations with 443 parity and age with ANOVA, therefore the PCA ultimately verified our individual ANOVA analyses.

444 Subsequently, we analyzed PC1 scores using ANOVA, which revealed a main effect of time (F(4, 445 37)=3.78, p=0.011; Fig. 10F), with higher scores at 13 months of age relative to all other age groups (all 446 p’s <0.033). Planned comparisons reveal a more robust age-related increase in PC1 scores in nulliparous 447 rats, with higher scores in 13- relative to 7- and 8-month-old rats (p’s<0.007). In contrast, there were no 448 significant differences in PC1 scores between any primiparous groups (p's>0.038; non-significant due to 449 Bonferroni correction). There was no significant main effect of reproductive status, nor an interaction 450 (p’s >0.11). Interestingly, the pattern observed here is akin to the age-related increase in IL-10, IFN- $\gamma$, 451 and IL-4 in nulliparous rats, obtained with individual ANOVA analyses. Further, IL-6 and TNF- $\alpha$ loaded 452 heavily onto PC2, and IL-1 $\beta$ and IL-5 loaded heavily onto PC3 and PC4, respectively (Table 1). 

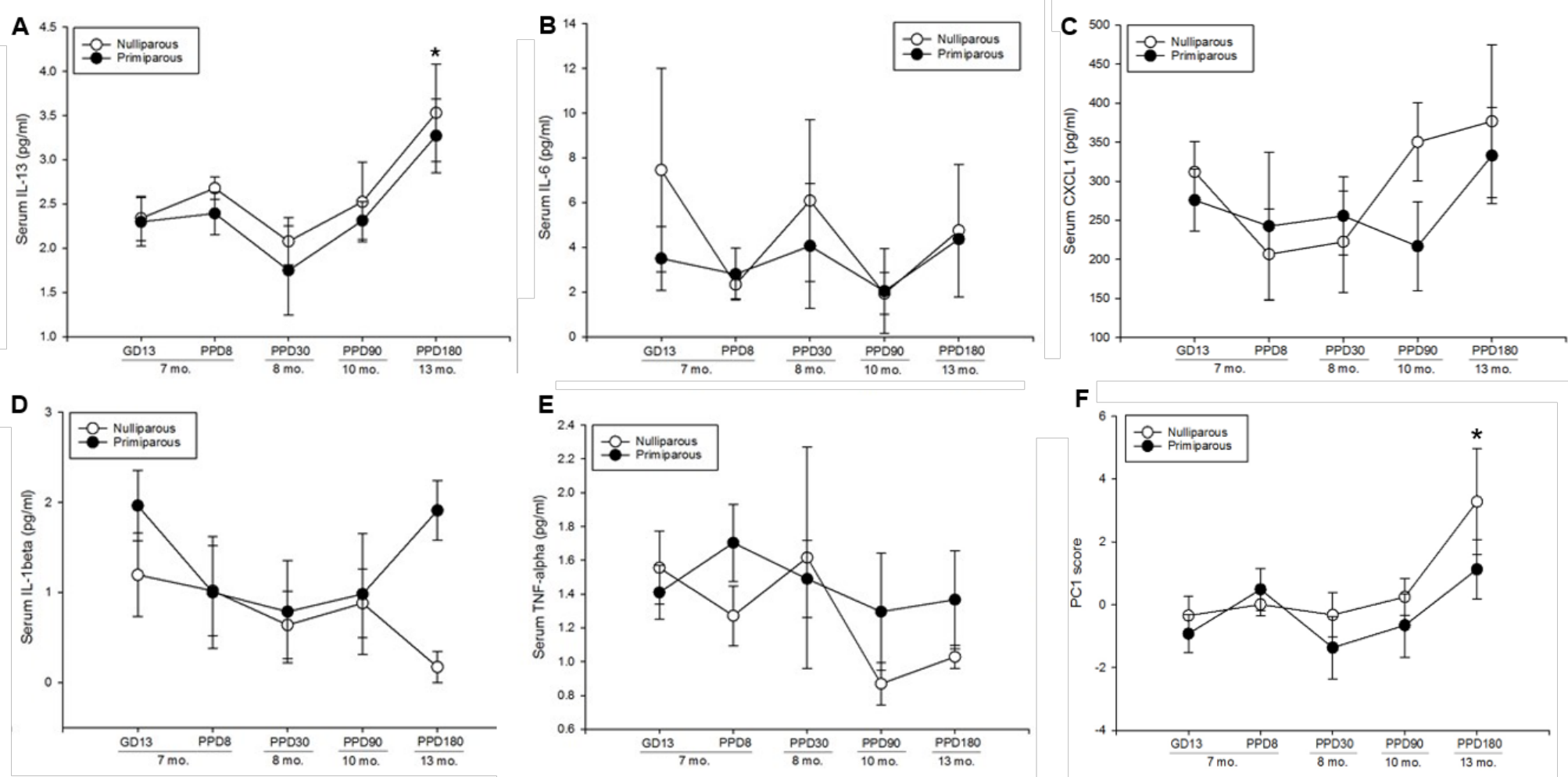

Figure 10. Serum concentrations of interleukin-13 (A), interleukin-6 (B), CXCL1 (C), interleukin- $\beta$ (D), and tumor necrosis factor alpha- $\alpha(\mathbf{E})$ in primiparous and nulliparous rats. The $\mathrm{x}$-axis represents time relative to gestation and parturition in primiparous rats, and approximate age in months. (A) There was a trend towards significance for serum IL-13 to increase with age regardless of reproductive status. * indicates $\mathrm{p}<0.002$ significantly different from 8-month old rats. Reproductive status and age had no significant effects on serum levels of interleukin-6 (B), CXCL1 (C), and interleukin- $\beta$ (D). (E) There were trends towards significance for parity to increase $(p=0.064)$ and for age to decrease $(p=0.061)$, TNF- $\alpha$ concentrations. (F) Principal Component 1 scores in primiparous and nulliparous rats, * indicates p's $<0.007$, significantly higher PC1 scores in 13- relative to 7- and 8-month-old nulliparous rats. Data are represented in mean values \pm SEM. GD= gestation day, PPD= postpartum day. Data are represented in mean values \pm SEM. IL-4= interleukin-4; IL-5 = interleukin-5; IL-13 = interleukin-13; GD= gestation day; PPD = postpartum day; mo.= age in months.

\subsection{Reproductive status influenced the direction and magnitude of correlations between microglia} and neurogenesis measures

455 Previous studies indicate that microglia play a role in adult hippocampal neurogenesis under baseline and 456 inflammatory conditions (Ekdahl et al., 2003; Monje, 2003; Sierra et al., 2010). However, the role of 457 microglia in motherhood-associated neurogenic changes have not been studied, thus, to indirectly examine 458 this we correlated our measures of neurogenesis and microglia. Increased average length of processes was 459 significantly correlated with a higher number of Ki67-IR cells in nulliparous $(r=0.58, p=0.005$; Fig. 460 11A), but not primiparous rats $(r=0.089, p=0.67)$, and these correlations were significantly different ( $\mathrm{z}$ $461=1.81, \mathrm{p}=0.035)$. Similarly, increased average length of processes was significantly correlated with a 
higher number of DCX-IR cells in nulliparous ( $r=0.62, p=0.002$; Fig. 11B), but not primiparous rats ( $r$ $463=0.11, p=0.60$; Fig. 11B $)$, and these correlations were significantly different $(z=1.96, p=0.025)$. 464 Interestingly, increased Ki-67-IR cell number was significantly associated with more DCX-IR cells in 465 nulliparous rats $(r=0.86, p<0.001$; Fig. 11C), but not in primiparous rats $(r=0.30, p=0.15$; Fig. 11C), 466 and the difference between the correlations was significant $(\mathrm{z}=3.04, \mathrm{p}=0.001)$. circulating cytokine concentrations and neural measures

469 Peripheral cytokine signals propagate to the brain and can influence neuroimmune function (Miller et al., 2014; Quan and Banks, 2007), thus we examined correlations between serum cytokine levels and microglial measures to assess whether central and peripheral inflammatory indicators are associated in parous and non-parous rats. Interestingly, in primiparous rats, the average length of Iba-1-IR cell processes was negatively correlated with serum IL-10 ( $r=-0.56$, p = 0.005; Fig. 11D), and IL-4 ( $\mathrm{r}=$ -

474 0.6, $p=0.002$; Fig. 11E). On the other hand, length of cell processes was not significantly correlated 475 with IL-10, or IL-4 in nulliparous rats (all p’s > 0.36). These correlations were significantly different 476 between reproductive status groups for IL-10 $(z=2.13, p=0.017)$, but not for IL-4 ( $\mathrm{p}=0.054)$.

477 Regardless of reproductive status, no other significant correlations were found between length of Iba-1478 IR cell processes and all other measured cytokines (all p's > 0.056; all trends towards significance 479 appear in primiparous groups only). Further, there were no significant correlations between any of the cytokines and Iba-1-IR cell density, regardless of reproductive status (all p’s > 0.23). IR cells (Ki67: -0.57, p=0.006; Fig. 11F; and DCX: $r=-0.70, p<0.001$; Fig. 11G). There were no significant correlations between any cytokines and Ki67- or DCX-IR cells in primiparous rats (all p’s > 0.19). These correlations were significantly different between reproductive status groups in both instances (DCX: $\mathrm{z}=-2.58, \mathrm{p}<0.005 ; \mathrm{Ki67:} \mathrm{z}=-1.82, \mathrm{p}=0.034)$.

\subsection{Serum 17 $\beta$-estradiol concentrations were not significantly correlated with hippocampal cell} proliferation, Iba-1 measures, or serum cytokine concentrations or nulliparous rats (p’s $>0.1$ ). Regardless of reproductive status, there were also no significant correlations between $17 \beta$-estradiol concentrations and any measure of Iba-1-IR cells (density, cell body size, average 

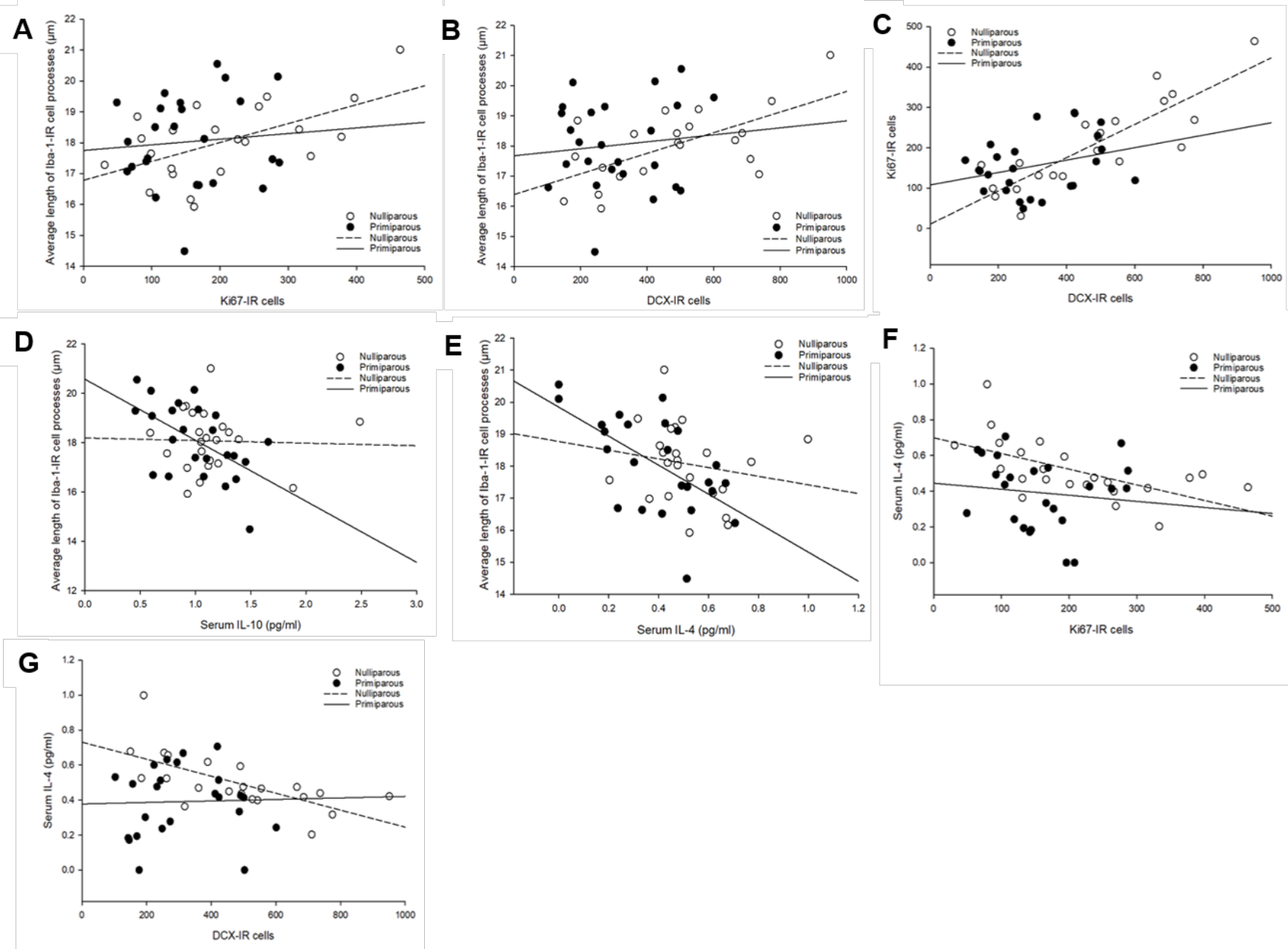

Figure 11. Correlations between dependent variables of interest. Increased average length of Iba-1-immunoreactive (IR) cell processes was associated with higher Ki67 (A) and doublecortin (DCX; B) expression in nulliparous rats only. KI67-IR cell number was significantly and positively correlated with DCX-IR cell number in nulliparous rats only (C). Elevated serum concentrations of IL-10 (D), and IL-4 (E) were significantly associated with shorter average Iba-1-IR cell processes in primiparous rats only. In nulliparous rats only, increased Ki67-IR (F) and DCXIR (G) cell number was significantly associated with lower serum IL-4.

\section{Discussion}

493 Here, we report short- and long-term effects of maternal experience on hippocampal neurogenesis, 494 microglial density and morphology in the dentate gyrus, and circulating cytokine levels, culminating six 495 months after parturition. We found that adult hippocampal neurogenesis was suppressed in midgestation and up to one month postpartum. Interestingly, the ageing trajectory of neurogenesis was modulated by reproductive experience, as neurogenesis levels declined from 7 to 13 months of age in nulliparous rats, but showed a slight increase in primiparous rats across the same period. Hippocampal cell proliferation was suppressed in mid-gestation and the early postpartum period in primiparous rats, 
500 but normalized thereafter, as an age-related decline in cell proliferation was observed regardless of

501 previous parity. Further, microglia in the dentate gyrus displayed a more activated morphology in the

502 early postpartum period, followed by a transient increase in microglial density in the later postpartum

503 period and overall smaller microglia soma size in primiparous compared to nulliparous rats. We found

504 alterations in circulating cytokine levels during pregnancy and the early postpartum period, and more

505 intriguingly, we show that the age-related changes in circulating cytokine levels were dependent on

506 parity. We also observed that reproductive status shifted the associations between microglia and

507 neurogenesis, with average length of Iba-1-IR cell processes being positively associated with

508 neurogenesis in nulliparous but not primiparous. Further, parity modulated the correlations between

509 serum cytokines and microglial morphology, and between serum cytokines and neurogenesis levels.

510 These correlations suggest that the relationships between immune processes and neurogenesis may be

511 modified with parity. Lastly, $17 \beta$-estradiol concentrations were reduced in the early postpartum period

512 (PPD8) and in mid-gestation (GD13), in line with previous findings (Rosenblatt et al., 1988). Beyond

513 the expected pregnancy and postpartum changes in circulating 17 $\beta$-estradiol, we found that

514 concentrations were not significant moderators of most variables, including hippocampal cell

515 proliferation, microglial density and morphology, and cytokine concentrations. Collectively our data

516 suggest that maternal experience has transient and delayed effects on hippocampal neurogenesis,

517 microglia, and the peripheral inflammatory milieu.

518 4.1. Adult hippocampal neurogenesis was suppressed during gestation and the postpartum period

519 We report that adult hippocampal neurogenesis, measured via the expression of doublecortin, was

520 suppressed beginning in mid-gestation in primigravid rats. Few studies to date have examined the

521 survival of new cells in the maternal hippocampus during gestation (Pawluski et al., 2010; Rolls et al.,

522 2008). Our findings are, however, consistent with a study that found suppressed neurogenesis in

523 pregnant mice during mid- and late-gestation (Rolls et al., 2008). In contrast, the expression of PSA-

524 NCAM was increased in the dentate gyrus of pregnant rats at GD18 (Banasr et al., 2001), indicating a

525 potential increase in neurogenesis, as PSA-NCAM is expressed on newly generated and migrating

526 neurons (Rutishauser, 2008). However, because PSA-NCAM is also expressed on neurons undergoing

527 other forms of plasticity (Rutishauser, 2008), its expression provides limited and non-specific

528 information regarding neurogenesis levels. Another study in rats found that the survival of cells

529 produced on gestation day 1 was not significantly altered when examined across gestation (Pawluski et

530 al., 2010), partially contrasting with our current findings. Importantly, however, DCX is expressed in

531 immature neurons between 2 hours and 21 days after production (Brown et al., 2003). Therefore, our 
532 current data provide information on the population of cells produced as early as 8 days prior to

533 impregnation, and as late as the day of euthanasia (GD13). Therefore, inconsistencies between the two

534 studies are not surprising, considering that the cell populations examined were produced under different

535 conditions. We also observe a concurrent reduction in cell proliferation on GD13 indicating that this

536 may underlie the decline in immature neurons at this time. Although no prior studies have examined

537 cell proliferation in mid-gestation, cell proliferation was not altered on GD1 (Pawluski et al., 2010),

538 GD7 (Shingo et al., 2003), or GD21 (Furuta and Bridges, 2005). Therefore, a more detailed time-course

539 analysis of hippocampal plasticity during pregnancy is warranted.

540 Consistent with a prior study (Workman et al., 2015), we also found reduced DCX expression in

541 the postpartum period, evident until PPD30. This finding is also in keeping with past work showing

542 reduced survival in new cells labelled on PPD2 and examined 21 days later (Pawluski and Galea, 2007),

543 and in new cells labelled in mid-gestation (GD11-12) and examined 14 days later, in the early postpartum

544 period (Rolls et al., 2008). We found reductions in hippocampal cell proliferation in the early postpartum

545 period, which normalized by PPD30, in line with previous data (Darnaudéry et al., 2007; Leuner et al.,

546 2007; Pawluski and Galea, 2007; Rolls et al., 2008). Thus, the suppression in immature neurons found at

547 PPD8 likely resulted from a reduction in both cell proliferation and survival, whereas the suppression at

548 PPD30 is likely due to decreased cell survival rather than proliferation. Although the functional

549 significance is not known, suppressed neurogenesis in the maternal brain may be mechanistically

550 associated with the enhanced susceptibility to mood disorders in the peripartum period (Hendrick et al.,

551 1998). Further, separate lines of evidence indicate that adult neurogenesis is involved in hippocampal

552 regulation of the HPA axis at least in males (Schloesser et al., 2009; Snyder et al., 2011), and that the HPA

553 axis undergoes substantial adaptations during pregnancy and the postpartum (De Weerth and Buitelaar,

554 2005; Lightman et al., 2001; Slattery and Neumann, 2008). Therefore, reductions in neurogenesis in the 555 maternal hippocampus could influence HPA axis function. Suppressed neurogenesis may also be linked 556 to deficits in hippocampus-dependent learning and memory reported in late pregnancy and the early 557 postpartum period (reviewed in (Workman et al., 2012)).

558 4.2. Maternal experience altered the trajectory of age-related changes in hippocampal neurogenesis

559 Between 8 and 13 months of age, immature neurons in the dentate gyrus declined significantly in 560 nulliparous rats but showed a slight increase in primiparous rats. We observe an age-related decline in 561 cell proliferation (Ki67-IR cells) regardless of reproductive status, suggesting that the differential effects 562 in immature neurons (DCX-IR cells) are driven by differences in cell survival. Previous work indicates 
563 that hippocampal neurogenesis steadily declines with age, with the most substantial decline occurring 564 between adulthood and middle age in female rats (Driscoll et al., 2006; Kuhn et al., 1996; Nacher et al., 565 2003), consistent with our current data from nulliparous rats. Thus, the increase in neurogenesis levels in 566 middle-aged primiparous rats suggests that reproductive experience can modify the trajectory of age567 related alterations in neurogenesis. Alternatively, it may also be reasonable to interpret this finding as 568 merely a normalization of neurogenesis to nulliparous levels. However, two previous reports indicate 569 higher neurogenesis levels in primiparous and multiparous relative to nulliparous middle-aged rats 570 (Barha et al., 2015; Galea et al., 2018), and as such an altered aging trajectory is conceivable. It is 571 possible that a more robust difference in neurogenesis levels would arise only after multiple 572 reproductive experiences or later into middle age. There is emerging evidence from human and rodent 573 studies suggesting that motherhood can alter the course of age-related cognitive decline (Beeri et al., 574 2009; Colucci et al., 2006; Cui et al., 2014; Gatewood et al., 2005). For example, reproductive 575 experience mitigated the age-related decline in spatial memory in rats (Gatewood et al., 2005) and mice 576 (Cui et al., 2014). Other studies indicate that parity is associated with cognitive impairment in the ageing 577 female (Beeri et al., 2009; Colucci et al., 2006). These inconsistencies may be reconciled by more 578 complex interactions with genetic factors that have been associated with pathological cognitive ageing 579 (Corbo et al., 2007; Cui et al., 2014). While speculative, the modest increase in hippocampal 580 neurogenesis in middle-aged primiparous rats may be associated with enhanced hippocampus-dependent 581 cognition that is seen at that time.

Interestingly, we observed differences in the relationship between levels of cell proliferation and immature neurons depending on reproductive status, such that a significant positive correlation between the two measures was only seen in nulliparous rats. In addition, increased IL-4 concentrations were associated with reduced proliferation and immature neurons in the hippocampus of nulliparous but not primiparous rats. Previous work points to a role of IL-4 in the regulation of cell proliferation under conditions of neurodegeneration (Bhattarai et al., 2016), therefore our findings suggest that this role may

588 be altered by parity. These relationships should be further investigated, as they appear when

589 reproductive status groups are collapsed across age, but nonetheless indicate that parity may modulate 590 the effects of immune signaling on hippocampal neurogenesis.

\subsection{Microglia assumed a de-ramified morphology in the early postpartum period}

592 Microglia display a predominantly ramified morphology under basal conditions, and de-ramification is 593 thought to be indicative of increased classical activation under inflammatory conditions (Luo and Chen, 
594 2012). Here, we show that microglia in the dentate gyrus exhibited significantly shortened processes at 8 595 days postpartum, suggesting an increase in microglial activation in the early postpartum period. This de596 ramification was a transient morphological modification as the average length of processes was not 597 significantly different from nulliparous controls by PPD30. We further show a small but significant shift 598 in the percentages of microglia assuming different morphological states at PPD8. Specifically, we 599 observe a reduction in ramified morphology and an increase in stout morphology, which in tandem with 600 our data on length of processes indicates a shift towards classical microglial activation in the dentate 601 gyrus in the early postpartum period. However, we interpret these findings with caution, as the 602 information that can be deduced from morphological phenotype about functional states is limited (Boche 603 et al., 2013). 17 $\beta$-estradiol concentrations significantly moderated the average length of microglial 604 processes, suggesting that the observed de-ramification at PPD8 might be moderated by postpartum 605 reductions in estradiol. However, as this measure of estradiol concentrations provides information about 606 a small window of time around perfusion, a complete picture of the role of estradiol cannot be 607 extrapolated from the current study. Future studies should also consider the role of other hormones, 608 including progesterone and corticosterone, in motherhood-associated changes microglia.

To our knowledge, only two studies to date have examined microglia in the maternal brain (Haim et al., 2017; Posillico and Schwarz, 2016), with findings partially consistent with our current data. Haim 611 et al. (2017) reported a decrease in the number of microglia with a ramified morphology on postpartum 612 day 8, in several regions including the dorsal hippocampus. This is in line with our current report of 613 microglial de-ramification and a shift in percentages of morphological states in the dentate gyrus on the 614 same postpartum day (PPD8). In the current study, we found an increase in the density of microglia in 615 the dorsal and ventral dentate gyrus at PPD30, but no alteration in density during gestation or the early 616 postpartum period. While no other studies have examined microglia in the maternal brain as late as 30 617 days postpartum, our finding that microglial density in the hippocampus remains unchanged during 618 gestation and the early postpartum period contrasts previous reports (Haim et al., 2017; Posillico and 619 Schwarz, 2016). These previous studies found reduced microglial density in several brain regions from 620 late gestation to the early postpartum (GD20, and PPD1, 8, and 21: Haim et al., 2017; PPD0: Posillico 621 and Schwarz, 2016). Further, Haim and colleagues (2017) found that microglial density normalized to 622 nulliparous control levels by PPD21 in all regions examined except the dorsal hippocampus. It appears, 623 however, that the inconsistencies in findings may be accounted for by differences in microglial densities 624 within sub-regions of the dentate gyrus, or by methodological differences related to density 625 measurement. For example, Haim et al. (2017) examined Iba-1 density within the dorsal dentate gyrus 
626 only, whereas we included samples from both the dorsal and ventral dentate gyrus. In addition, as we 627 were primarily interested in the neurogenic niche, we quantified Iba-1-IR cells within the GCL, the 628 SGZ, and a thin band of the ML, whereas Haim and colleagues did not specify sub-regions within the 629 dentate gyrus. Finally, Haim et al (2017) utilized optical density, whereas density here was defined as 630 the number of cells per volume of dentate gyrus. Interestingly, the increase in microglial density that we 631 find at PPD30 coincides with a return to normalized microglial morphology. We speculate that this may 632 represent a resolution from the pro-inflammatory state at PPD8. Overall, our novel data provide an 633 important addition to the literature indicating that pregnancy-related immune adaptations are not limited 634 to the periphery, but also exist in the brain. More specifically, our data indicate the existence of a pro635 inflammatory hippocampal environment in the early postpartum period. Importantly, increased 636 microglial activation may be central to the pathophysiology of depression (Kreisel et al., 2014; Miller 637 and Raison, 2015; Setiawan et al., 2015), thus it is conceivable that similar processes are implicated in 638 postpartum depression. Our current findings which point to an increase in microglial activation in the 639 early postpartum may represent a neural mechanism of enhanced susceptibility to mood disorders at that time, however additional work would be required to directly test this.

642 Specifically, although the number of microglial cell processes increased significantly with age in 643 nulliparous rats, this effect was prevented by parity. Further, parity reduced microglial soma size, an 644 effect which appears to emerge at PPD30 onwards. The functional significance of these alterations 645 cannot be determined from the current study, but as increased microglial soma size is indicative of 646 classical activation, it is possible that parity may dampen microglial activation in the ageing brain. To 647 gain better insight into the functional significance of these changes, future studies should investigate 648 how previous parity may impact microglial structure and function in the ageing brain in response to an 649 immune challenge.

Interestingly, we found that reproductive status affected the associations between microglia and neurogenesis in the hippocampus. Specifically, increased cell proliferation and immature neurons were 652 significantly associated with more ramified microglial morphology in nulliparous rats only. On the other 653 hand, increased cell proliferation and immature neurons were associated with higher microglial density 654 in primiparous rats only. These observations suggest that the neuroimmune regulation of adult 655 hippocampal neurogenesis is affected by reproductive status. Importantly, in addition phagocytic activity 656 during development and disease, microglia are important players in the regulation of adult hippocampal 657 neurogenesis, where they phagocytose apoptotic new cells, while maintaining ramified morphology and 
658 a non-inflammatory environment (Sierra et al., 2010). Thus, future research should directly examine the 659 possibilities of microglial phagocytosis in the regulation of neurogenesis during pregnancy and the 660 postpartum period.

\section{4.5. Maternal experience modifies the age-related changes in circulating cytokine levels}

662 In addition to expected cytokine alterations during pregnancy and the early postpartum period (Holtan et 663 al., 2015; Shimaoka et al., 2000), we observed both persistent and delayed effects of reproductive 664 experience on the circulating cytokine profile. Specifically, after an initial increase at PPD8, IL-4 levels 665 were persistently blunted in primiparous rats relative to nulliparous controls. Further, in nulliparous but 666 not primiparous rats, IFN- $\gamma$ and IL-10 increased and IL-5 declined significantly with age. Adaptations to 667 the immune systems during pregnancy and the early postpartum period are well established (PrabhuDas 668 et al., 2015). On the other hand, little attention has been paid to potential long-term effects of 669 motherhood on the immune system. To our knowledge, only a few studies have examined the effect of 670 parity on immune systems in aged female mice and one in aged rats. Together these studies suggested 671 that parity may delay certain indicators of immune senescence, as they relate to alterations in cytokine 672 production in vitro from activated spleen cells (Barrat et al., 1997a), and the distribution of immune cell 673 populations in the spleen (Barrat et al., 1997b), and bone marrow (Barrat et al., 1999). Another study 674 from our laboratory found a trend for increased serum levels of IL-6 in primiparous compared to 675 nulliparous rats at 15 months of age (Galea et al., 2018). Here, we report that a single reproductive 676 experience alters serum cytokine levels when examined in middle age, up to six months after the 677 reproductive event.

Importantly, peripheral cytokines can affect brain function, as they can access the central nervous system through various mechanisms, including active and passive transport, and the activation of cytokine receptors on afferent nerve fibers (reviewed in Miller et al., 2014; Quan and Banks, 2007). Thus, the effects of parity to modify age-related changes in peripheral cytokines may have ramifications 682 for the ageing brain in general, and more specifically the hippocampus, as it contains one of the highest 683 densities of proinflammatory cytokine receptors in the brain (reviewed in Loftis et al., 2010).

684 Inflammation is a core characteristic of the ageing processes, and the pro-inflammatory cytokine IFN- $\gamma$ 685 increases with age (Oxenkrug, 2011; Rodríguez et al., 2007). Thus, our current data suggest that parity may prevent or delay at least certain aspects of ageing-related inflammation. Interestingly, from PPD30 onwards, we observed a sustained suppression in serum IL-4 in primiparous rats relative to nulliparous controls. Traditionally considered an anti-inflammatory cytokine (Hart et al., 1989), elevated IL-4 in 
nulliparous rats may be suggestive of a compensatory response to attenuate a pro-inflammatory state that is indicated by elevated IFN- $\gamma$. However, IL-4 is pleiotropic (Milner et al., 2010), and as such also can

691 have pro-inflammatory properties. For example, sustained exposure to elevated levels of IL-4 was 692 associated with increased inflammation (Milner et al., 2010). The same study found prolonged IL-4 693 exposure to be associated specifically with elevated levels of IL-10 and IFN- $\gamma$, but not IL-6 and TNF- $\alpha$.

694 This is in line with our current data in which IL-4, IL-10, and IFN- $\gamma$ were concurrently elevated in 695 middle-aged nulliparous rats. Thus, the cytokine profile in nulliparous groups may be alternatively 696 driven by an increase in IL-4. We investigated correlations between peripheral cytokines and microglial 697 morphology as a plethora of work suggests that systemic inflammation can profoundly affect microglial 698 activation (Reviewed in Hoogland et al., 2015). Interestingly, we found that higher concentrations of IL69910 and IL-4 were associated with more de-ramified microglial morphology in primiparous but not 700 nulliparous rats. The mechanisms and consequences of these altered relationships between peripheral 701 cytokines and microglial morphology are not known, and these findings should be interpreted with 702 caution, as the correlations were detected when age was not included as a factor. In the future, it is also 703 important to examine whether parity may have similar effects on age-related changes in brain cytokines, 704 particularly in the hippocampus. While the functional consequences of the observed differences in 705 cytokine profiles cannot be determined from our current finding, we demonstrate here that the trajectory 706 of immune senescence is altered by parity.

\section{5. Conclusions}

708 In summary, we report that maternal experience suppressed hippocampal neurogenesis (proliferation and 709 immature neurons) during gestation and the postpartum period and mitigated the decline in neurogenesis 710 in middle age (immature neurons). Maternal experience also resulted in transient microglial de-

711 ramification in the dentate gyrus, suggesting the existence of a pro-inflammatory hippocampal

712 environment in the early postpartum period. In addition to short-term cytokine alterations, maternal 713 experience modified the trajectory of age-related changes in circulating cytokine levels. These findings

714 should encourage future work aimed at delineating the functional consequences for behaviour and 715 immune function across the peripartum period and beyond, especially in relation to maternal mood and 716 cognition. Importantly, our data provide support for the notion that female reproductive history should 717 be regarded as an important determinant of ageing-related changes in physiology.

\section{Acknowledgments}

719 The Authors thank Dr. Timothy Kieffer and Travis Webber for generously providing access to their 
720 Sector Imager, and Arianne Albert for her assistance with principal component analyses.

\section{Funding}

722 This work was supported by a grant from the Canadian Institute of Health Research to LAMG

723 (PJT148662), and a Four-Year Doctoral Fellowship from the University of British Columbia to RM.

\section{Conflicts of Interest}

725 The authors declare no conflicts of interest. 
Table 1. Mean number of Iba-1-IR cells used for process length analyses \pm standard error of the mean. There were no significant differences between groups.

\begin{tabular}{ll}
\hline Group & Number of Iba-1-IR cells analyzed \\
\hline Nulliparous - 7 mo. & $23.40 \pm 1.03$ \\
Nulliparous - 7.5 mo. & $21.00 \pm 2.04$ \\
Nulliparous - 8 mo. & $20.80 \pm 2.13$ \\
Nulliparous - 10 mo. & $21.20 \pm 1.16$ \\
Nulliparous - 13 mo. & $22.25 \pm 2.32$ \\
Primiparous - GD13 & $23.67 \pm 1.67$ \\
Primiparous - PPD8 & $22.00 \pm 0.77$ \\
Primiparous - PPD30 & $23.60 \pm 1.47$ \\
Primiparous - PPD90 & $20.60 \pm 1.03$ \\
Primiparous - PPD180 & $23.20 \pm 0.37$ \\
\hline
\end{tabular}

Table 2. Mean percentage of proliferative, intermediate, and post-mitotic doublecortin (DCX)immunoreactive (IR) cells in the granule cell layer \pm standard error of the mean. Parity and age did not significantly affect the maturational stage of DCX-IR cells.

\begin{tabular}{llll}
\hline Group & \% Proliferative & \% Intermediate & \% Post-mitotic \\
\hline Nulliparous - 7 mo. & $37.60 \pm 1.60$ & $17.20 \pm 2.15$ & $45.20 \pm 2.65$ \\
Nulliparous - 7.5 mo. & $26.40 \pm 2.86$ & $18.80 \pm 1.85$ & $54.80 \pm 2.87$ \\
Nulliparous - 8 mo. & $35.60 \pm 4.71$ & $20.80 \pm 1.02$ & $46.40 \pm 4.45$ \\
Nulliparous - 10 mo. & $29.60 \pm 6.52$ & $21.60 \pm 2.64$ & $48.80 \pm 4.84$ \\
Nulliparous - 13 mo. & $25.33 \pm 8.74$ & $20.00 \pm 1.15$ & $54.67 \pm 8.97$ \\
Primiparous - GD13 & $32.00 \pm 6.23$ & $18.40 \pm 2.93$ & $49.60 \pm 5.84$ \\
Primiparous - PPD8 & $28.40 \pm 5.31$ & $16.00 \pm 1.10$ & $56.40 \pm 6.71$ \\
Primiparous - PPD30 & $27.60 \pm 4.26$ & $17.20 \pm 0.80$ & $55.20 \pm 4.88$ \\
Primiparous - PPD90 & $23.60 \pm 3.19$ & $16.80 \pm 3.01$ & $59.60 \pm 4.53$ \\
Primiparous - PPD180 & $28.40 \pm 5.71$ & $16.00 \pm 2.45$ & $56.00 \pm 6.23$ \\
\hline
\end{tabular}

Table 3. Principal Component Analysis loading table.

\begin{tabular}{lllll}
\hline & PC1 & PC2 & PC3 & PC4 \\
\hline IFN- $\gamma$ & $\mathbf{0 . 8 7}$ & -0.28 & 0.01 & -0.04 \\
IL-10 & $\mathbf{0 . 9 1}$ & -0.15 & -0.12 & 0.027 \\
IL-13 & $\mathbf{0 . 8 7}$ & -0.18 & 0.27 & 0.12 \\
IL-1ß & -0.06 & 0.25 & $\mathbf{0 . 7 8}$ & -0.46 \\
IL-4 & $\mathbf{0 . 8 9}$ & -0.17 & -0.05 & 0.03 \\
IL-5 & -0.24 & 0.10 & 0.30 & $\mathbf{0 . 8 7}$ \\
IL-6 & 0.46 & $\mathbf{0 . 7 7}$ & -0.11 & 0.091 \\
CXCL1 & 0.41 & 0.46 & 0.42 & 0.11 \\
TNF- $\alpha$ & 0.25 & $\mathbf{0 . 7 7}$ & -0.39 & -0.096 \\
\hline
\end{tabular}




\section{References}

727 Aghaeepour, N., Ganio, E.A., Mcilwain, D., Tsai, A.S., Tingle, M., Van Gassen, S., Gaudilliere, D.K., Baca, Q., McNeil, L., Okada, R., Ghaemi, M.S., Furman, D., Wong, R.J., Winn, V.D., Druzin, M.L., El-Sayed, Y.Y., Quaintance, C., Gibbs, R., Darmstadt, G.L., Shaw, G.M., Stevenson, D.K.,

Altman, J., Das, G.D., 1965. Autoradiographic and histological evidence of postnatal hippocampal Tibshirani, R., Nolan, G.P., Lewis, D.B., Angst, M.S., Gaudilliere, B., 2017. An immune clock of

Banasr, M., Hery, M., Brezun, J.M., Daszuta, A., 2001. Serotonin mediates oestrogen stimulation of cell proliferation in the adult dentate gyrus. Eur. J. Neurosci. 14, 1417-1424. doi:10.1046/j.0953816X.2001.01763.X

Barha, C.K., Galea, L.A.M., 2011. Motherhood alters the cellular response to estrogens in the hippocampus later in life. Neurobiol. Aging 32, 2091-2095.

Barha, C.K., Hanna, C.W., Salvante, K.G., Wilson, S.L., Robinson, W.P., Altman, R.M., Nepomnaschy, P.A., 2016. Number of children and telomere length in women: A prospective, longitudinal evaluation. PLoS One 11. doi:10.1371/journal.pone.0146424

Barha, C.K., Lieblich, S.E., Chow, C., Galea, L.A.M., 2015. Multiparity-induced enhancement of hippocampal neurogenesis and spatial memory depends on ovarian hormone status in middle age.

Barrat, F., Lesourd, B., Boulouis, H.J., Thibault, D., Vincent-Naulleau, S., Gjata, B., Louise, A., Neway,

Barrat, F., Lesourd, B.M., Louise, A., Boulouis, H.J., Vincent-Naulleau, S., Thibault, D., Sanaa, M., T., Pilet, C., 1997a. Sex and parity modulate cytokine production during murine ageing. Clin. Exp.

Barrat, F.S., Lesourd, B.M., Louise, A.S., Boulouis, H.J., Thibault, D.J., Neway, T., Pilet, C.A., 1999. 
Pregnancies modulate B lymphopoiesis and myelopoiesis during murine ageing. Immunology 98, 604-611. doi:10.1046/j.1365-2567.1999.00918.x

756

757

758

759

760

761

762

763

764

765

766

767

768

769

770

771

772

773

774

775

776

777

778

779

780

781

Beeri, M.S., Rapp, M., Schmeidler, J., Reichenberg, A., Purohit, D.P., Perl, D.P., Grossman, H.T., Prohovnik, I., Haroutunian, V., Silverman, J.M., 2009. Number of children is associated with neuropathology of Alzheimer's disease in women. Neurobiol. Aging 30, 1184-1191. doi:10.1016/j.neurobiolaging.2007.11.011

Bennett, H.A., Einarson, A., Taddio, A., Koren, G., Einarson, T.R., 2004. Prevalence of depression during pregnancy: systematic review. Obs. Gynecol 103, 698-709. doi:10.1097/01.AOG.0000116689.75396.5f

Bhattarai, P., Thomas, A.K., Cosacak, M.I., Papadimitriou, C., Mashkaryan, V., Froc, C., Reinhardt, S., Kurth, T., Dahl, A., Zhang, Y., Kizil, C., 2016. IL4/STAT6 Signaling Activates Neural Stem Cell Proliferation and Neurogenesis upon Amyloid- $\beta 42$ Aggregation in Adult Zebrafish Brain. Cell Rep. 17, 941-948. doi:10.1016/j.celrep.2016.09.075

Boche, D., Perry, V.H., Nicoll, J.A.R., 2013. Review: Activation patterns of microglia and their identification in the human brain. Neuropathol. Appl. Neurobiol. doi:10.1111/nan.12011

Bodnar, T.S., Taves, M.D., Lavigne, K.M., Woodward, T.S., Soma, K.K., Weinberg, J., 2017. Differential activation of endocrine-immune networks by arthritis challenge: Insights from colonyspecific responses. Sci. Rep. 7, 1-14. doi:10.1038/s41598-017-00652-4

Boldrini, M., Fulmore, C.A., Tartt, A.N., Simeon, L.R., Pavlova, I., Poposka, V., Rosoklija, G.B., Stankov, A., Arango, V., Dwork, A.J., Hen, R., Mann, J.J., 2018. Human Hippocampal Neurogenesis Persists throughout Aging. Cell Stem Cell 589-599. doi:10.1016/j.stem.2018.03.015

Bridges, R.S., 2015. Neuroendocrine regulation of maternal behavior. Front. Neuroendocrinol. doi:10.1016/j.yfrne.2014.11.007

Brown, J.P., Couillard-Després, S., Cooper-Kuhn, C.M., Winkler, J., Aigner, L., Kuhn, H.G., 2003. Transient Expression of Doublecortin during Adult Neurogenesis. J. Comp. Neurol. 467, 1-10. doi:10.1002/cne.10874

Campbell, S., MacQueen, G., 2004. The role of the hippocampus in the pathophysiology of major depression. [Review] [121 refs]. J. Psychiatry \&amp; Neurosci. 29, 417-426. 
Catalano, R.D., Lannagan, T.R.M., Gorowiec, M., Denison, F.C., Norman, J.E., Jabbour, H.N., 2010. Prokineticins: Novel mediators of inflammatory and contractile pathways at parturition? Mol. Hum. Reprod. doi:10.1093/molehr/gaq014

785

Colucci, M., Cammarata, S., Assini, A., Croce, R., Clerici, F., Novello, C., Mazzella, L., Dagnino, N., Mariani, C., Tanganelli, P., 2006. The number of pregnancies is a risk factor for Alzheimer’s disease. Eur. J. Neurol. 13, 1374-1377. doi:10.1111/j.1468-1331.2006.01520.x

Corbo, R.M., Gambina, G., Ulizzi, L., Monini, P., Broggio, E., Rosano, A., Scacchi, R., 2007. Combined effect of apolipoprotein e genotype and past fertility on age at onset of Alzheimer's disease in women. Dement. Geriatr. Cogn. Disord. 24, 82-85. doi:10.1159/000103866

Cui, J., Jothishankar, B., He, P., Staufenbiel, M., Shen, Y., Li, R., 2014. Amyloid precursor protein mutation disrupts reproductive experience-enhanced normal cognitive development in a mouse model of alzheimer's disease. Mol. Neurobiol. 49, 103-112. doi:10.1007/s12035-013-8503-x

Cuttler, C., Graf, P., Pawluski, J.L., Galea, L. a M., 2011. Everyday life memory deficits in pregnant women. Can. J. Exp. Psychol. 65, 27-37. doi:10.1037/a0022844

Darcy, J.M., Grzywacz, J.G., Stephens, R.L., Leng, I., Clinch, C.R., Arcury, T.A., 2011. Maternal Depressive Symptomatology: 16-Month Follow-up of Infant and Maternal Health-Related Quality of Life. J. Am. Board Fam. Med. 24, 249-257. doi:10.3122/jabfm.2011.03.100201

Darnaudéry, M., Perez-Martin, M., Del Favero, F., Gomez-Roldan, C., Garcia-Segura, L.M., Maccari, S., 2007. Early motherhood in rats is associated with a modification of hippocampal function. Psychoneuroendocrinology 32, 803-812. doi:10.1016/j.psyneuen.2007.05.012

De Groot, R.H., Vuurman, E.F., Hornstra, G., Jolles, J., 2006. Differences in cognitive performance during pregnancy and early motherhood. Psychol. Med. 36, 1023-1032. doi:10.1017/S0033291706007380

De Weerth, C., Buitelaar, J.K., 2005. Physiological stress reactivity in human pregnancy - A review, in: Neuroscience and Biobehavioral Reviews. pp. 295-312. doi:10.1016/j.neubiorev.2004.10.005

Driscoll, I., Howard, S.R., Stone, J.C., Monfils, M.H., Tomanek, B., Brooks, W.M., Sutherland, R.J., 2006. The aging hippocampus: A multi-level analysis in the rat. Neuroscience 139, 1173-1185. doi:10.1016/j.neuroscience.2006.01.040 
810 Dulac, C., O’Connell, L.A., Wu, Z., 2014. Neural control of maternal and paternal behaviors. Science (80-. ). 345, 765-770. doi:10.1126/science.1253291

812 Ekdahl, C.T., Claasen, J.-H., Bonde, S., Kokaia, Z., Lindvall, O., 2003. Inflammation is detrimental for 813 neurogenesis in adult brain. Proc. Natl. Acad. Sci. 100, 13632-13637.

814 doi:10.1073/pnas.2234031100

815 Eriksson, P.S., Perfilieva, E., Björk-Eriksson, T., Alborn, A.M., Nordborg, C., Peterson, D.A., Gage, 816 F.H., 1998. Neurogenesis in the adult human hippocampus. Nat. Med. 4, 1313-7. doi:10.1038/3305

817 Furuta, M., Bridges, R.S., 2005. Gestation-induced cell proliferation in the rat brain. Brain Res Dev $818 \quad$ Brain Res 156, 61-66.

819 Galea, L.A.M., Leuner, B., Slattery, D.A., 2014. Hippocampal plasticity during the peripartum period: 820 Influence of sex steroids, stress and ageing. J. Neuroendocrinol. doi:10.1111/jne.12177

821 Galea, L.A.M., Ormerod, B.K., Sampath, S., Kostaras, X., Wilkie, D.M., Phelps, M.T., 2000. Spatial 822 Working Memory and Hippocampal Size across Pregnancy in Rats. Horm. Behav. 37, 86-95. 823 doi:10.1006/hbeh.1999.1560

824 Galea, L.A.M., Roes, M.M., Dimech, C.J., Chow, C., Mahmoud, R., Lieblich, S.E., Duarte-Guterman, 825 P., 2018. Premarin has opposing effects on spatial learning, neural activation, and serum cytokine 826 levels in middle-aged female rats depending on reproductive history. Neurobiol. Aging 70, 291827 307. doi:10.1016/j.neurobiolaging.2018.06.030

828 Gatewood, J.D., Morgan, M.D., Eaton, M., McNamara, I.M., Stevens, L.F., MacBeth, A.H., Meyer, 829 E.A.A., Lomas, L.M., Kozub, F.J., Lambert, K.G., Kinsley, C.H., 2005. Motherhood mitigates 830 aging-related decrements in learning and memory and positively affects brain aging in the rat. Brain 831 Res. Bull. 66, 91-98. doi:10.1016/j.brainresbull.2005.03.016

832 Groer, M.E., Jevitt, C., Ji, M., 2015. Immune Changes and Dysphoric Moods Across the Postpartum. 833 Am. J. Reprod. Immunol. 73, 193-198. doi:10.1111/aji.12322

834 Guerreiro, R.J., Santana, I., Brás, J.M., Santiago, B., Paiva, A., Oliveira, C., 2007. Peripheral 835 inflammatory cytokines as biomarkers in Alzheimer’s disease and mild cognitive impairment. 836 Neurodegener. Dis. 4, 406-412. doi:10.1159/000107700 
837 Haim, A., Julian, D., Albin-Brooks, C., Brothers, H.M., Lenz, K.M., Leuner, B., 2017. A survey of 838 neuroimmune changes in pregnant and postpartum female rats. Brain. Behav. Immun. 59, 67-78. doi:10.1016/j.bbi.2016.09.026

Hall, M.E., George, E.M., Granger, J.P., 2011. The Heart During Pregnancy. Rev. Española Cardiol. (English Ed. 64, 1045-1050. doi:10.1016/j.rec.2011.07.008

842

843

844

845

846

Hart, P.H., Vitti, G.F., Burgess, D.R., Whitty, G.A., Piccoli, D.S., Hamilton, J.A., 1989. Potential antiinflammatory effects of interleukin 4: suppression of human monocyte tumor necrosis factor alpha, interleukin 1, and prostaglandin E2. Proc. Natl. Acad. Sci. 86, 3803-3807. doi:10.1073/pnas.86.10.3803

Helle, S., Lummaa, V., Jokela, J., 2004. Accelerated immunosenescence in preindustrial twin mothers. Proc. Natl. Acad. Sci. U. S. A. 101, 12391-12396. doi:10.1073/pnas.0402215101

Hendrick, V., Altshuler, L.L., Suri, R., 1998. Hormonal Changes in the Postpartum and Implications for Postpartum Depression. Psychosomatics 39, 93-101. doi:10.1016/S0033-3182(98)71355-6

Hodes, G.E., Pfau, M.L., Leboeuf, M., Golden, S.A., Christoffel, D.J., Bregman, D., Rebusi, N., Heshmati, M., Aleyasin, H., Warren, B.L., Labonté, B., Horn, S., Lapidus, K.A., Stelzhammer, V., Wong, E.H.F., Bahn, S., Krishnan, V., Bolaños-Guzman, C.A., Murrough, J.W., Merad, M., Russo, S.J., 2014. Individual differences in the peripheral immune system promote resilience versus susceptibility to social stress. Proc. Natl. Acad. Sci. 111, 16136-16141. doi:10.1073/pnas.1415191111

Hoekzema, E., Barba-Müller, E., Pozzobon, C., Picado, M., Lucco, F., García-García, D., Soliva, J.C., Tobeña, A., Desco, M., Crone, E.A., Ballesteros, A., Carmona, S., Vilarroya, O., 2016. Pregnancy leads to long-lasting changes in human brain structure. Nat. Neurosci. doi:10.1038/nn.4458

Holtan, S.G., Chen, Y., Kaimal, R., Creedon, D.J., Enninga, E.A.L., Nevala, W.K., Markovic, S.N., 2015. Growth modeling of the maternal cytokine milieu throughout normal pregnancy: Macrophage-derived chemokine decreases as inflammation/counterregulation increases. J. Immunol. Res. 2015. doi:10.1155/2015/952571

Hoogland, I.C.M., Houbolt, C., van Westerloo, D.J., van Gool, W.A., van de Beek, D., 2015. Systemic inflammation and microglial activation: systematic review of animal experiments. J. 
Neuroinflammation 12, 114. doi:10.1186/s12974-015-0332-6

866 Karperien, A., Ahammer, H., Jelinek, H.F., 2013. Quantitating the subtleties of microglial morphology with fractal analysis. Front. Cell. Neurosci. doi:10.3389/fncel.2013.00003

868 869

Kinsley, C.H., Madonia, L., Gifford, G.W., Tureski, K., Griffin, G.R., Lowry, C., Williams, J., Collins, J., McLearie, H., Lambert, K.G., 1999. Motherhood improves learning and memory. Nature 402, 137-138. doi:10.1038/45957

Korzhevskii, D.E., Kirik, O. V., 2016. Brain Microglia and Microglial Markers. Neurosci. Behav. Physiol. 46, 284-290. doi:10.1007/s11055-016-0231-z

Kreisel, T., Frank, M.G., Licht, T., Reshef, R., Ben-Menachem-Zidon, O., Baratta, M. V, Maier, S.F., Yirmiya, R., 2014. Dynamic microglial alterations underlie stress-induced depressive-like behavior and suppressed neurogenesis. Mol. Psychiatry 19, 699-709. doi:10.1038/mp.2013.155

Kuhn, H.G., Dickinson-Anson, H., Gage, F.H., 1996. Neurogenesis in the dentate gyrus of the adult rat: age-related decrease of neuronal progenitor proliferation. J Neurosci 16, 2027-2033. doi:0270-6474

Lain, K.Y., Catalano, P.M., 2007. Metabolic changes in pregnancy. Clin. Obstet. Gynecol. 50, 938-948. doi:10.1097/GRF.0b013e31815a5494

Lee, J., Lee, Y., Yuk, D., Choi, D., Ban, S., Oh, K., Hong, J., 2008. Neuro-inflammation induced by lipopolysaccharide causes cognitive impairment through enhancement of beta-amyloid generation. J. Neuroinflammation 5, 37. doi:10.1186/1742-2094-5-37

Leuner, B., Christian, M., Liron, N., Elizabeth, G., 2007. Maternal Experience Inhibits the Production of Immature Neurons in the Hippocampus During the Postpartum Period Through. Hippocampus 17, 434-442. doi:10.1002/hipo

Leuner, B., Gould, E., 2010. Dendritic growth in medial prefrontal cortex and cognitive flexibility are enhanced during the postpartum period. J. Neurosci. 30, 13499-503. doi:10.1523/JNEUROSCI.3388-10.2010

Lightman, S.L., Windle, R.J., Wood, S.A., Kershaw, Y.M., Shanks, N., Ingram, C.D., 2001. Peripartum plasticity within the hypothalamo-pituitary-adrenal axis, in: Progress in Brain Research. pp. 111129. doi:10.1016/S0079-6123(01)33009-1 
892 Loftis, J.M., Huckans, M., Morasco, B.J., 2010. Neuroimmune mechanisms of cytokine-induced 893 depression: Current theories and novel treatment strategies. Neurobiol. Dis.

894 doi:10.1016/j.nbd.2009.11.015

895 896

897

898

899

900

901

902

903

904

905

906

907

908

909

910

911

912

913

914

915

916

917

Louveau, A., Harris, T.H., Kipnis, J., 2015. Revisiting the Mechanisms of CNS Immune Privilege. Trends Immunol. doi:10.1016/j.it.2015.08.006

Luo, X.-G., Chen, S.-D., 2012. The changing phenotype of microglia from homeostasis to disease. Transl. Neurodegener. 1, 9. doi:10.1186/2047-9158-1-9

Mahmoud, R., Wainwright, S.R., Chaiton, J.A., Lieblich, S.E., Galea, L.A.M., 2016a. Ovarian hormones, but not fluoxetine, impart resilience within a chronic unpredictable stress model in middle-aged female rats. Neuropharmacology 107, 278-293. doi:10.1016/j.neuropharm.2016.01.033

Mahmoud, R., Wainwright, S.R., Galea, L.A.M., 2016b. Sex hormones and adult hippocampal neurogenesis: Regulation, implications, and potential mechanisms. Front. Neuroendocrinol. 41, 129-152. doi:10.1016/j.yfrne.2016.03.002

Miller, A.H., Haroon, E., Raison, C.L., Felger, J.C., 2014. Cytokine Targets in the Brain: Impact on Neurotransmitters and Neurocircuits Andrew. Depress Anxiety 30, 297-306. doi:10.1146/annurevcellbio-092910-154240.Sensory

Miller, A.H., Raison, C.L., 2015. The role of inflammation in depression: from evolutionary imperative to modern treatment target. Nat. Rev. Immunol. 16, 22-34. doi:10.1038/nri.2015.5

Milner, J.D., Orekov, T., Ward, J.M., Cheng, L., Torres-Velez, F., Junttila, I., Sun, G., Buller, M., Morris, S.C., Finkelman, F.D., Paul, W.E., 2010. Sustained IL-4 exposure leads to a novel pathway for hemophagocytosis, inflammation, and tissue macrophage accumulation. Blood 116, 2476-2483. doi:10.1182/blood-2009-11-255174

Monje, M.L., 2003. Inflammatory Blockade Restores Adult Hippocampal Neurogenesis. Science (80-. ). 302, 1760-1765. doi:10.1126/science.1088417

Mor, G., Cardenas, I., 2010. The Immune System in Pregnancy: A Unique Complexity. Am. J. Reprod. Immunol. doi:10.1111/j.1600-0897.2010.00836.X 
919 Nacher, J., Alonso-Llosa, G., Rosell, D.R., McEwen, B.S., 2003. NMDA receptor antagonist treatment 920 increases the production of new neurons in the aged rat hippocampus. Neurobiol Aging 24, 273921 284. doi:S0197458002000969 [pii]

922 Nimmerjahn, A., Kirchhoff, F., Helmchen, F., 2005. Neuroscience: Resting microglial cells are highly 923 dynamic surveillants of brain parenchyma in vivo. Science (80-. ). doi:10.1126/science.1110647

924 O’Hara, M.W., 2009. Postpartum depression: What we know. J. Clin. Psychol. doi:10.1002/jclp.20644

925 Oatridge, A., Holdcroft, A., Saeed, N., Hajnal, J. V., Puri, B.K., Fusi, L., Bydder, G.M., 2002. Change in 926 brain size during and after pregnancy: Study in healthy women and women with preeclampsia. Am. 927 J. Neuroradiol. 23, 19-26.

928 Overstreet-Wadiche, L.S., Bromberg, D. a, Bensen, A.L., Westbrook, G.L., 2006. Seizures accelerate 929 functional integration of adult-generated granule cells. J. nNeuroscience.

930 doi:10.1523/JNEUROSCI.5508-05.2006

931 Oxenkrug, G.F., 2011. Interferon-gamma-inducible kynurenines/pteridines inflammation cascade:

932 Implications for aging and aging-associated psychiatric and medical disorders. J. Neural Transm. 933 118, 75-85. doi:10.1007/s00702-010-0475-7

934 Parkhurst, C.N., Yang, G., Ninan, I., Savas, J.N., Yates, J.R., Lafaille, J.J., Hempstead, B.L., Littman, 935 D.R., Gan, W.B., 2013. Microglia promote learning-dependent synapse formation through brain936 derived neurotrophic factor. Cell 155, 1596-1609. doi:10.1016/j.cell.2013.11.030

937 Pawluski, J.L., Barakauskas, V.E., Galea, L.A.M., 2010. Pregnancy decreases oestrogen receptor alpha 938 expression and pyknosis, but not cell proliferation or survival, in the hippocampus. J.

939 Neuroendocrinol. 22, 248-257. doi:10.1111/j.1365-2826.2010.01960.x

940 Pawluski, J.L., Galea, L.A.M., 2007. Reproductive experience alters hippocampal neurogenesis during 941 the postpartum period in the dam. Neuroscience 149, 53-67.

942 doi:10.1016/j.neuroscience.2007.07.031

943 Pawluski, J.L., Galea, L.A.M., 2006. Hippocampal morphology is differentially affected by reproductive 944 experience in the mother. J. Neurobiol. 66, 71-81. doi:10.1002/neu.20194

945 Pawluski, J.L., Walker, S.K., Galea, L.A.M., 2006. Reproductive experience differentially affects spatial 
reference and working memory performance in the mother. Horm. Behav. 49, 143-149.

948

949

950

951

952

953

954

955

956

957

958

959

960

961

962

963

964

965

966

967

968

969

970

971

972

973 doi:10.1016/j.yhbeh.2005.05.016

Plümpe, T., Ehninger, D., Steiner, B., Klempin, F., Jessberger, S., Brandt, M., Römer, B., Rodriguez, G.R., Kronenberg, G., Kempermann, G., 2006. Variability of doublecortin-associated dendrite maturation in adult hippocampal neurogenesis is independent of the regulation of precursor cell proliferation. BMC Neurosci. 7, 77. doi:10.1186/1471-2202-7-77

Posillico, C.K., Schwarz, J.M., 2016. An investigation into the effects of antenatal stressors on the postpartum neuroimmune profile and depressive-like behaviors. Behav. Brain Res. 298, 218-228. doi:10.1016/j.bbr.2015.11.011

PrabhuDas, M., Bonney, E., Caron, K., Dey, S., Erlebacher, A., Fazleabas, A., Fisher, S., Golos, T., Matzuk, M., McCune, J.M., Mor, G., Schulz, L., Soares, M., Spencer, T., Strominger, J., Way, S.S., Yoshinaga, K., Loreau, M., Naeem, S., Inchausti, P., 2015. Immune mechanisms at the maternalfetal interface: perspectives and challenges. Nat. Immunol. 16, 237-243. doi:10.1038/ni.3131.Immune

Quan, N., Banks, W.A., 2007. Brain-immune communication pathways. Brain. Behav. Immun. doi:10.1016/j.bbi.2007.05.005

Rannevik, G., Carlström, K., Jeppsson, S., Bjerre, B., Svanberg, L., 1986. A prospective long-term study in women from pre-menopause to post-menopause: changing profiles of gonadotrophins, oestrogens and androgens. Maturitas. doi:10.1016/0378-5122(86)90038-1

Rodríguez, M.I., Escames, G., López, L.C., López, A., García, J.A., Ortiz, F., Acuña-Castroviejo, D., 2007. Chronic melatonin treatment reduces the age-dependent inflammatory process in senescenceaccelerated mice. J. Pineal Res. 42, 272-279. doi:10.1111/j.1600-079X.2006.00416.x

Rolls, A., Schori, H., London, A., Schwartz, M., 2008. Decrease in hippocampal neurogenesis during pregnancy: a link to immunity. Mol. Psychiatry 13, 468-469. doi:10.1038/sj.mp.4002126

Rosenblatt, J.S., Mayer, A.D., Giordano, A.L., 1988. Hormonal basis during pregnancy for the onset of maternal behavior in the rat. Psychoneuroendocrinology. doi:10.1016/0306-4530(88)90005-4

Rossant, J., Cross, J.C., 2001. PLACENTAL DEVELOPMENT: LESSONS FROM MOUSE MUTANTS. Nat. Rev. Genet. 2, 538-548. doi:10.1038/35080570 
974 Rutishauser, U., 2008. Polysialic acid in the plasticity of the developing and adult vertebrate nervous system. Nat. Rev. Neurosci. 9, 26-35. doi:10.1038/nrn2285

Sahay, A., Hen, R., 2007. Adult hippocampal neurogenesis in depression. Nat. Neurosci. 10, 1110-5. doi:10.1038/nn1969

Schloesser, R.J., Manji, H.K., Martinowich, K., 2009. Suppression of adult neurogenesis leads to an increased hypothalamo-pituitary-adrenal axis response. Neuroreport 20, 553-557. doi:10.1097/WNR.0b013e3283293e59

Scholzen, T., Gerdes, J., 2000. The Ki-67 protein: From the known and the unknown. J. Cell. Physiol.

983

984

985

986

987

988

989

990

991

992

993

994

995

996

997

998

999

Schumacher, A., Costa, S.D., Zenclussen, A.C., 2014. Endocrine factors modulating immune responses in pregnancy. Front. Immunol. doi:10.3389/fimmu.2014.00196

Schwarz, J.M., Sholar, P.W., Bilbo, S.D., 2012. Sex differences in microglial colonization of the developing rat brain. J. Neurochem. doi:10.1111/j.1471-4159.2011.07630.x

Setiawan, E., Wilson, A.A., Mizrahi, R., Rusjan, P.M., Miler, L., Rajkowska, G., Suridjan, I., Kennedy, J.L., Rekkas, P.V., Houle, S., Meyer, J.H., 2015. Role of Translocator Protein Density, a Marker of Neuroinflammation, in the Brain During Major Depressive Episodes. JAMA Psychiatry 72, 268. doi:10.1001/jamapsychiatry.2014.2427

Shimaoka, Y., Hidaka, Y., Tada, H., Amino, N., Nakamura, T., Murata, Y., Mitsuda, N., Morimoto, Y., 2000. Changes in cytokine production during and after normal pregnancy. Am J Reprod Immunol 44, 143-147.

Shingo, T., Gregg, C., Enwere, E., Fujikawa, H., Hassam, R., Geary, C., Cross, J.C., Weiss, S., 2003. Pregnancy-stimulated neurogenesis in the adult female forebrain mediated by prolactin. Science 299, 117-20. doi:10.1126/science.1076647

Sierra, A., Beccari, S., Diaz-Aparicio, I., Encinas, J.M., Comeau, S., Tremblay, M.È., 2014. Surveillance, phagocytosis, and inflammation: How never-resting microglia influence adult hippocampal neurogenesis. Neural Plast. doi:10.1155/2014/610343

1000 Sierra, A., Encinas, J.M., Deudero, J.J.P., Chancey, J.H., Enikolopov, G., Overstreet-Wadiche, L.S., 
Tsirka, S.E., Maletic-Savatic, M., 2010. Microglia shape adult hippocampal neurogenesis through apoptosis-coupled phagocytosis. Cell Stem Cell 7, 483-495. doi:10.1016/j.stem.2010.08.014

1003 Slattery, D.A., Neumann, I.D., 2008. No stress please! Mechanisms of stress hyporesponsiveness of the 1004 maternal brain. J. Physiol. 586, 377-385. doi:10.1113/jphysiol.2007.145896

1005 Snyder, J.S., Soumier, A., Brewer, M., Pickel, J., Cameron, H.A., 2011. Adult hippocampal neurogenesis buffers stress responses and depressive behaviour. Nature 476, 458-461. doi:10.1038/nature10287

1008 Sweatt, J.D., 2004. Hippocampal function in cognition. Psychopharmacology (Berl). 174. doi:10.1007/s00213-004-1795-9

vom Berg, J., Prokop, S., Miller, K.R., Obst, J., Kälin, R.E., Lopategui-Cabezas, I., Wegner, A., Mair, F., Schipke, C.G., Peters, O., Winter, Y., Becher, B., Heppner, F.L., 2012. Inhibition of IL-12/IL23 signaling reduces Alzheimer’s disease-like pathology and cognitive decline. Nat. Med. 18, 1812-1819. doi:10.1038/nm.2965

1014 Workman, J.L., Barha, C.K., Galea, L.A.M., 2012. Endocrine substrates of cognitive and affective 1015 changes during pregnancy and postpartum. Behav. Neurosci. 126, 54-72. doi:10.1037/a0025538

1016 Workman, J.L., Raineki, C., Weinberg, J., Galea, L.A.M., 2015. Alcohol and pregnancy: Effects on 1017 maternal care, HPA axis function, and hippocampal neurogenesis in adult females. 1018 Psychoneuroendocrinology 57, 37-50. doi:10.1016/j.psyneuen.2015.03.001

1019 Yau, S., Li, A., So, K., 2015. Involvement of Adult Hippocampal Neurogenesis in 2015. 